\author{
A. Moya · J. Christensen-Dalsgaard - S. Charpinet - Y. Lebreton · A. Miglio * \\ J. Montalbán · M.J.P.F.G. Monteiro · J. Provost · I.W. Roxburgh · R. Scuflaire · \\ J.C.Suárez · M. Suran
}

\title{
Inter-comparison of the $\mathrm{g}$-, $\mathrm{f}$ - and $\mathrm{p}$-modes calculated using different oscillation codes for a given stellar model
}

Received: date / Accepted: date

\begin{abstract}
In order to make asteroseismology a powerful tool to explore stellar interiors, different numerical codes should give the same oscillation frequencies for the same input physics. Any differences found when comparing the numerical values of the eigenfrequencies will be an important piece of information regarding the numerical structure of the code. The ESTA group was created to analyze the non-physical sources of these differences. The work presented in this report is a part of Task 2 of the ESTA group. Basically the work is devoted to test, compare and, if needed, optimize the seismic codes used to calculate the eigenfrequencies to be finally compared with observations. The first step in this comparison is presented here. The oscillation codes of nine research groups in the field have been used in this study. The same physics has been imposed for all the codes in order to isolate the non-physical dependence of any possi-
\end{abstract}

A. Moya · J.C. Suárez

Instituto de Astrofísica de Andalucía- CSIC

Cno. Bajo de Huetor, 50, Granada, Spain

E-mail: moya@iaa.es

J. Christensen-Dalsgaard

Institut for Fysik og Astronomi, og Dansk AsteroSeismisk Center, Aarhus Universitet, Denmark

S. Charpinet

Observatoire Midi-Pyrénées, France

Y. Lebreton

Observatoire de Paris, GEPI, CNRS UMR 8111, Meudon, France

A. Miglio $\cdot$ J. Montalbán $\cdot$ R. Scuflaire

Institut d'Astrophysique et Geophysique, Université de Liège, Belgium

M.J.P.F.G. Monteiro

Centro de Astrofísica da Universidade do Porto and Departamento de Matemática Aplicada da Faculdade de Ciências, Universidade do Porto, Portugal

J. Provost

Observatoire de la Cote d'Azur, Nice, France

I. Roxburgh

Astronomy Unit, Queen Mary, University of London, UK

M. Suran

Astronomical Institute of the Romanian Academy, Romania ble difference. Two equilibrium models with different grids, 2172 and 4042 mesh points, have been used, and the latter model includes an explicit modelling of semiconvection just outside the convective core. Comparing the results for these two models illustrates the effect of the number of mesh points and their distribution in particularly critical parts of the model, such as the steep composition gradient outside the convective core. A comprehensive study of the frequency differences found for the different codes is given as well. These differences are mainly due to the use of different numerical integration schemes. The number of mesh points and their distribution are crucial for interpreting the results. The use of a second-order integration scheme plus a Richardson extrapolation provides similar results to a fourth-order integration scheme. The proper numerical description of the Brunt-Väisälä frequency in the equilibrium model is also critical for some modes. This influence depends on the set of the eigenfunctions used for the solution of the differential equations. An unexpected result of this study is the high sensitivity of the frequency differences to the inconsistent use of values of the gravitational constant $(G)$ in the oscillation codes, within the range of the experimentally determined ones, which differ from the value used to compute the equilibrium model. This effect can provide differences for a given equilibrium model substantially larger than those resulting from the use of different codes or numerical techniques; the actual differences between the values of $G$ used by the different codes account for much of the frequency differences found here.

Keywords Stars - Stellar oscillations · Numerical solution

PACS 97.10.Sj $\cdot 97.10 . \mathrm{Cv} \cdot 97.90 .+\mathrm{j}$

\section{Introduction}

Asteroseismology is at present being developed as an efficient instrument in the study of stellar interiors and evolution. Pulsational frequencies are the most important asteroseismic observational inputs. It is evident that a meaningful analysis of the observation, in terms of the basic physics of 
stellar interiors which is the ultimate target of the investigation, requires reliable computation of oscillation frequencies for specified physics. This is a two-step process, involving first the computation of stellar evolutionary models and secondly the computation of frequencies for the resulting models. Lebreton et al. (this volume) provide an overview of the tests of stellar model calculations. Here we consider the computation of the oscillation frequencies.

An evident goal is that the computed frequencies, for a given model, should have errors well below the observational error, which in the case of the CoRoT mission is expected to be below $0.1 \mu \mathrm{Hz}$ (Baglin et al., 2006). For the Kepler mission (e.g., Christensen-Dalsgaard et al., 2007), with expected launch in early 2009, selected stars may be observed continuously for several years and errors as low as $10^{-3} \mu \mathrm{Hz}$ may be reachable, particularly for modes excited by the heat-engine mechanism. Since errors resulting from numerical problems are typically highly systematic, they may affect the asteroseismic inferences even if they are substantially below the random errors in the observed frequencies. This must be kept in mind in the assessment of the estimates of the numerical errors.

During the last decades a lot of codes obtaining numerical solutions of an adiabatic system of differential equations describing stellar oscillations have been developed. In order to ascertain whether any possible difference in the description of the same observational data by different numerical codes is due to physical descriptions or to different numerical integration schemes, the inter-comparison of these oscillation codes in a fixed and homogeneous framework is absolutely necessary. Some effort has been already done in the past but only regarding pairs of codes. Some codes have also developed a lot of internal precision tests. However, there is a lack of inter-comparison of a large enough set of codes. We aim in this study try to fix a set of minimum requirements for a code to be sure that any difference found is only due to a different physical assumption.

Ideally, for a given model there should be a set of 'true' frequencies with which the results of the different codes could be compared. This ideal situation could probably be approximated by considering polytropic models for which it is relatively straightforward to calculate the equilibrium structure with essentially arbitrary accuracy (see also Christensen-Dalsgaard and Mullan, 1994). In practice, the situation for realistic stellar models is more complex. Owing to the complexity of the stellar evolution calculation the models are often available on a numerical mesh which is not obviously adequate for the pulsation calculation. The effect of this on the frequency computation depends on the detailed formulation of the equations in the pulsation codes. These formulations are equivalent only if the equilibrium model satisfies the 'dynamical' equations of stellar structure, i.e., the mass equation and the equation of hydrostatic support, and this is obviously not exactly true for a model computed on a finite (possibly even relatively sparse) mesh. One might define a consistent set of frequencies for a given model by interpolating it onto a very dense mesh and resetting it to ensure that the relevant equations of stellar structure are satisfied. The model is fully characterized by the dependence on distance $r$ to the centre of density $\rho$ and the adiabatic exponent $\Gamma_{1}=(\partial \ln P / \partial \ln \rho)_{\text {ad }}, P$ being pressure and the derivative being at constant specific entropy. Thus one could interpolate $\rho(r)$ and $\Gamma_{1}(r)$ to a fine mesh, and recompute the mass distribution and pressure by integrating the mass equation and the equation of hydrostatic equilibrium. Frequencies of this model should then be essentially independent of the formulation of the oscillation equation and would provide a suitable reference with which to compare other frequency calculations. Such a test may be carried out at a later stage in the ESTA effort.

In Task 2, for now, we aim at testing, comparing and optimizing the seismic codes used to calculate the oscillations of existing models of different types of stars. In order to do so we consider steps in the comparison by addressing some of the most relevant items that must be compared regarding the seismic characterization of the models:

- Step 1: comparison of the frequencies from different seismic codes for the same model.

- Step 2: comparison of the frequencies from the same seismic code for different models of the same stellar case provided by different equilibrium codes.

- Step 3: comparison of the frequencies for specific pulsators.

The work presented here is mostly focused on step 1 . At this step three different equilibrium models have been used. Two of them have been computed using CESAM (Morel and Lebreton, 2007), with 902 and 2172 mesh points, and a third one with 4042 mesh points provided by ASTEC (Christensen-Dalsgaard, 2007).We present intercomparisons using the two models with the larger numbers of mesh points. The same physics and physical constants (except $G$ ) are used for all the oscillation codes. Frequencies in the range of $[20,2500] \mu \mathrm{Hz}$, belonging to spherical degrees $\ell=0,1,2$ and 3 have been calculated, in order to recover most of the possible values we can find in the observational photometric data.

We present in Sect. 2 the equilibrium models used and we analyze their main features. Section 3 is devoted to the different oscillation codes used, discussing common requirements and variations in the treatment. In Sect. 4 the direct comparison of frequencies for different frequency ranges and spherical degrees is presented in detail. Sections 5 and 6 analyze the same inter-comparison for the values of the large and small separations, respectively. Section 7 discusses the dominant effects that contribute to the differences in frequencies determined by the different codes. Conclusions are given in Sect. 8.

\section{The equilibrium models.}

To ensure that any difference obtained in the inter-comparisons is only due to differences in the numerical schemes, 
Table 1 General characteristics of the models used for the intercomparison.

\begin{tabular}{lllllll}
\hline$M / M_{\odot}$ & $\log T_{\text {eff }}$ & $\begin{array}{l}\text { Age } \\
\text { (in My) }\end{array}$ & $X_{\mathrm{c}}$ & $R / R_{\odot}$ & $\begin{array}{l}\text { Mesh } \\
\text { points }\end{array}$ & $\begin{array}{l}G \\
{\left[10^{-8} \mathrm{cgs}\right]}\end{array}$ \\
\hline 1.5 & 3.826 & 1355 & 0.4 & 1.731 & 4042 & 6.6716823 \\
1.5 & 3.830 & 1368 & 0.4 & 1.724 & 2172 & 6.67232 \\
\hline
\end{tabular}

we imposed to all the codes the use of the same equilibrium models. These models were supplied in several formats: OSC, FGONG, SROX, and FAMDL. The first model was required to have 900 mesh points, and it was provided by CESAM. The differences in this case reached unacceptable values of $2-3 \mu \mathrm{Hz}$ when the same integration schemes are compared, and even $10 \mu \mathrm{Hz}$ when the use or not of the Richardson extrapolation are compared. The maximum difference found for large separations with this model is $1 \mu \mathrm{Hz}$. This showed that either a larger number of mesh points or Richardson extrapolation was necessary 1

The second model was also provided by CESAM (referred from now on as M2k). It uses a grid, with 2172 mesh points, more suitable for asteroseismic purposes. General characteristics of the model are presented in Table 1. These are typical of a $\gamma$ Doradus star showing oscillations in the asymptotic g-mode regime, and also around the fundamental radial mode. A priori, solar-like pulsations cannot be excluded for this type of star and therefore it can be a good candidate for a global study. In Fig. 1, $A^{*}$ (which is a quantity directly related to the Brunt-Väisälä frequency $N^{2}$ : $A^{*}=r g^{-1} N^{2}$, where $g$ is the gravitational acceleration) is depicted as a function of the relative radius $(x=r / R)$ in a region of steeply varying hydrogen abundance, and hence mean molecular weight $\mu$, just outside the convective core. The model is in a phase of a growing convective core. If diffusion and settling are neglected this leads to a discontinuity in the hydrogen abundance and hence, formally, to a delta function in $A^{*}$; also, there is a region of 'semiconvection' at the edge of the core. In fact, the figure shows that three points in this model display an erratic variation in $A^{*}$ just in the transition region between the convective and the radiative zone; also, the mesh resolution of this region of rapid variation seems inadequate. This is emphasized by Fig. 2 which shows the distribution of mesh points of this model along the stellar radius, indicating that there are not enough mesh points in this transition zone. As discussed below, these features of model M2k give rise to frequency differences in the comparison, particularly for those modes for which this inner part is critical for their physical description.

The third and last model is a 4042 mesh points model (from now on M4k) provided by ASTEC. General characteristics of the model are presented in Table 1. It has been computed to have overall characteristics similar to the previous CESAM model in order to understand better the differences. However, as discussed by Christensen-Dalsgaard (this vol-

1 Detailed results of this investigation can be found at http://www. astro.up.pt/corot/compfreqs/task2/

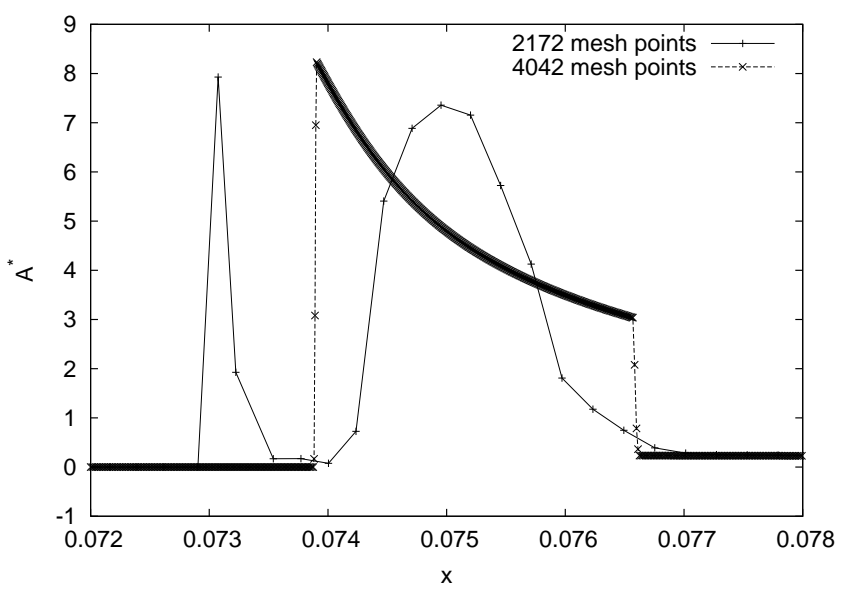

Fig. $1 A^{*}$ (related to the Brunt-Väisälä frequency) as a function of the relative radius for the two equilibrium models discussed in the text in the $\mu$-gradient zone, close to the convective core. The mesh points provided in the models are indicated.

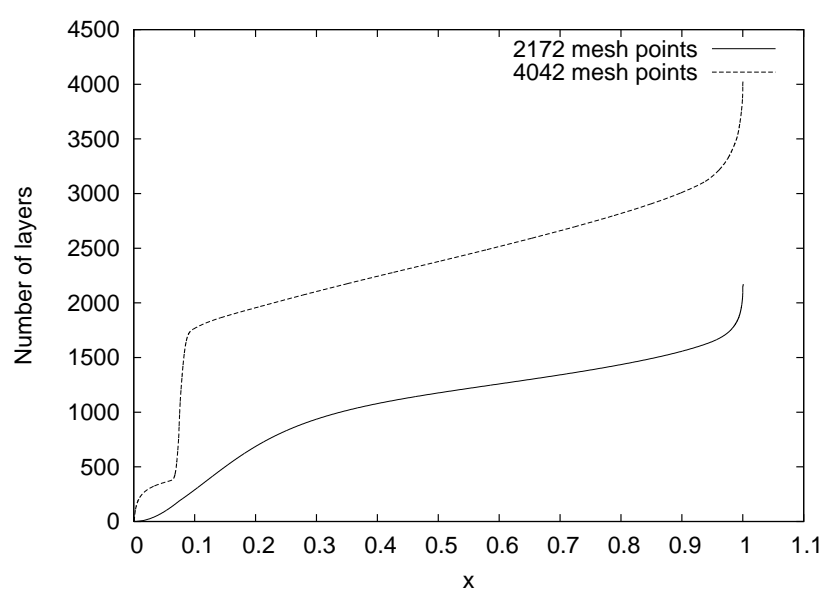

Fig. 2 Accumulated number of layers as a function of the relative radius for the two equilibrium models explained in the text.

ume) particular care has been taken in the treatment of the $\mu$-gradient region; the semiconvective region was replaced by a region with a steep gradient in the hydrogen abundance, defined such as to ensure neutral stability of the temperature gradient. As a result, $A^{*}$ for this model, also presented in Fig. 1, shows a well-defined and reasonable behaviour. The distribution of the mesh points can be found in Fig. 2. In the central and outer parts of the stellar model, the distribution is similar to the CESAM model M2k. It is in the inner zones, particularly in the boundary region between the convective core and the $\mu$-gradient zone, that the models present different mesh-point distributions, with M4k providing a far superior resolution of this critical region. 


\section{Oscillation codes and requirements.}

All oscillation codes involved in this task were asked to provide adiabatic frequencies in the range of $[20,2500] \mu \mathrm{Hz}$ and spherical degrees $\ell=0,1,2$ and 3 . In addition, the solution of the equations must satisfy the following requirements:

- To use the mesh provided by the equilibrium model, no re-meshing is allowed.

- To set the Lagrangian perturbation to the pressure to zero $(\delta P=0)$ as outer mechanical boundary condition.

- To use the physical constants prescribed in Task 1.

- To use linear adiabatic equations.

Nevertheless, some other schemes for the numerical solution of the differential equations (from now on called for simplicity "degrees of freedom") remain open. Nine oscillation codes of different research groups in the field have been used in this inter-comparison exercise. A summary of the participating codes and the different "degrees of freedom" provided by each one is found in Table 2 and include:

- Set of eigenfunctions: Use of the Lagrangian or the Eulerian perturbation to the pressure $\left(\delta P\right.$ or $\left.P^{\prime}\right)$. This obviously affects the form of the equations; in particular, when using $\delta P$ the equations do not depend explicitly on $A^{*}$.

- Order of the integration scheme: Most of the codes use a second-order scheme, but some of them have implemented a fourth-order scheme.

- Richardson extrapolation: Some of the codes using a second-order scheme have the possibility to use Richardson extrapolation (Shibahashi et al., 1981) to decrease the truncation error; combining a second-order scheme with Richardson extrapolation yields errors scaling as $\mathscr{N}^{-4}, \mathscr{N}$ being the number of mesh points (e.g. Christensen-Dalsgaard and Mullan, 1994).

- Integration variable: Two integration variables are used: $1)$ the radius $(r)$, or 2$)$ the ratio $r / P$. The latter variable may reduce the effect of rounding errors in the outer layers (see Sect.7.3).

- Despite the requirement that the physical constants be fixed at the values for Task 1, the codes used slightly different values of the gravitational constant $G$, as listed in Table 2. Ideally the equilibrium model should have been computed with the prescribed Task 1 value $(6.6716823 \times$ $10^{-8} \mathrm{cgs}$ ) which should then have been used for the oscillation calculations. In practice Model M4k was computed with $G=6.67232 \times 10^{-8} \mathrm{cgs}$. Using different values of $G$ in the oscillation equations clearly gives rise to inconsistencies, with potential effects on the frequencies, as discussed further in Sect.7.1.

Note that most of the oscillation codes put $\ell=0$ in the general non-radial differential equations to obtain the radial modes, except for LOSC that uses the LAWE differential equation (Linear Adiabatic Wave Equation for radial modes), and for GraCo for which results will be shown for both sets of equations. It should be noted that the LAWE

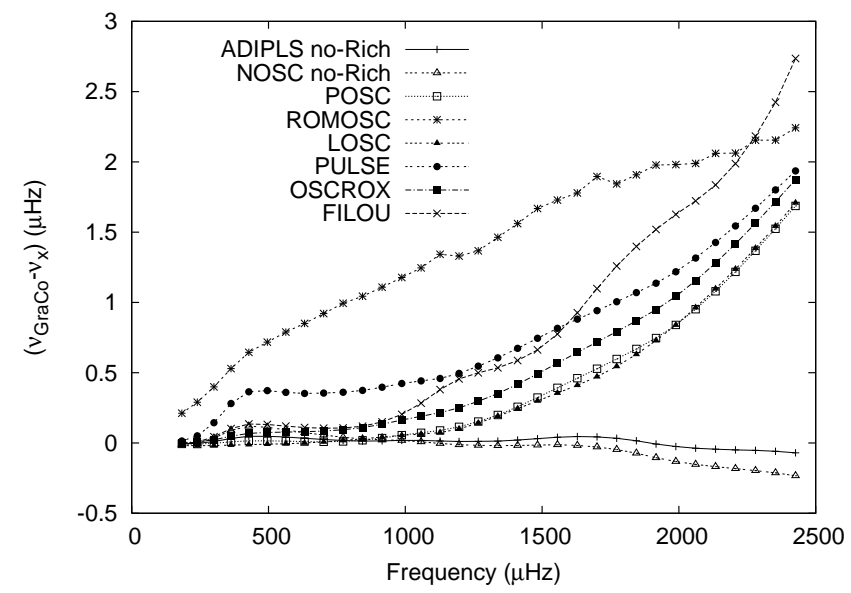

Fig. 3 Frequency comparison (reference line is $\mathrm{GraCo}$ ) for modes with $\ell=0$ obtained for the model M2k. ADIPLS and NOSC frequencies have been obtained without using the Richardson extrapolation.

does not depend on $A^{*}$. All the main characteristics and numerical schemes are presented in previous chapters of this volume.

The Nice code (NOSC) has the options of using $P^{\prime}$ or $\delta P$ as dependent variables, and $r$ or $r / P$ as independent variable. However, all NOSC results presented here use $P^{\prime}$ and $r$.

The use of the mesh provided in the equilibrium model, rather than meshes optimized for the different kinds of modes, may result in inadequate resolution of the rapid variation of high-order $\mathrm{p}$ - and g-modes and hence larger truncation errors in the solution of the differential equations. In these cases, therefore, the second-order scheme may result in unacceptable errors. Here the use of higher-order integration schemes (a fourth-order scheme or a second-order scheme followed by Richardson extrapolation) is therefore expected to give better results. For low- and intermediateorder modes we expect little effect of the use of higher-order schemes.

\section{Frequency inter-comparisons.}

In this section, the results of the direct frequency inter-comparison are presented. We have structured this study splitting the frequency range in three parts for the non-radial case, only one for the radial case, and comparing codes only with similar selections of "degrees of freedom". In addition, the influence of using different selections in the same code (GraCo in this case) is shown; for these tests the value of $G$ in the oscillation calculations was the same as was used to compute the equilibrium model.

\subsection{Radial modes.}

These are shown in a single frequency range. In Fig. 3 the results obtained using the model M2k are presented. 
Table 2 List of participating codes in this inter-comparison and "degrees of freedom" for each code: (a) Eigenfunctions, (b) Order of the Integration Scheme (2 or 4), (c) Use (y) or not (n) of the Richardson extrapolation, (d) integration variable used, and (e) the choice of the gravitational constant $G$. The references for each code are also given.

\begin{tabular}{lllllll}
\hline Code & E.F. & I.S. & Rich. & I.V. & $\begin{array}{l}G \\
{\left[10^{-8} \mathrm{cgs}\right]}\end{array}$ & Reference \\
\hline ADIPLS & $P^{\prime}$ & 2 & $\mathrm{y}, \mathrm{n}$ & $r$ & 6.67232 & Christensen-Dalsgaard (2007) \\
FILOU & $P^{\prime}$ & 2 & $\mathrm{n}$ & $r$ & 6.67232 & Suarez and Goupil (2007) \\
GraCo & $P^{\prime}, \delta P$ & 2 & $\mathrm{y}, \mathrm{n}$ & $r, r / P$ & 6.6716823 & Moya and Garrido (2007) \\
LOSC & $\delta P$ & 4 & $\mathrm{n}$ & $r$ & 6.67232 & Scuflaire et al. (2007) \\
NOSC & $P^{\prime}, \delta P$ & 2 & $\mathrm{y}, \mathrm{n}$ & $r, r / P$ & 6.67259 & Provost (2007) \\
OSCROX & $P^{\prime}$ & 4 & $\mathrm{n}$ & $r$ & 6.6716823 & Roxburgh (2007) \\
POSC & $P^{\prime}$ & 2 & $\mathrm{y}, \mathrm{n}$ & $r$ & 6.6716823 & Monteiro (2007) \\
PULSE & $P^{\prime}$ & 4 & $\mathrm{n}$ & $r / P$ & 6.6716823 & Brassard and Charpinet (2007) \\
LNAWENR & $P^{\prime}$ & 2 & $\mathrm{n}$ & $r$ & 6.67232 & Suran (2007) \\
\hline
\end{tabular}

The reference line for all the inter-comparisons is selected to be GraCo. In this figure the reference frequencies have been obtained using $P^{\prime}$, second order, no Richardson extrapolation and $r$ as independent variable (see Table 2). Two sets of codes can be identified in the figure, ADIPLSNOSC-GraCo, with differences lower than $0.25 \mu \mathrm{Hz}$, and all with the same "degrees of freedom", and OSCROX-PULSELOSC-POSC with differences for high frequencies lower than $2 \mu \mathrm{Hz}$ with $\mathrm{GraCo}$, but with differences among them around $0.5 \mu \mathrm{Hz}$. This second set of codes differs from the first one in the use of a fourth-order numerical scheme instead of a second-order one. In the present figure, in Fig. [5 and in Figs 7 and 8 , showing inter-comparisons with model M2k, POSC has been chosen as representative of the codes using second order plus Richardson extrapolation (see Table 2). We can see how the use of this integration procedure provides similar results as the fourth-order solutions. These differences, around $0.5 \mu \mathrm{Hz}$ for codes using the same integration scheme, and $2 \mu \mathrm{Hz}$ for codes using different schemes, are larger than the expected precision of the coming observational data. Therefore this effect can change any detailed physical description as interpreted by different oscillation codes. Also, we point out that the differences between the codes using fourth-order schemes and GraCo results using a second-order scheme indicate that the model has an insufficient number of mesh points for asteroseismic studies.

Figure 4 shows the results obtained for the model M4k. In the top panel models with second order, no Richardson extrapolation, $r$, are depicted. All the differences are lower in magnitude than $0.014 \mu \mathrm{Hz}$, i.e., two orders of magnitude lower than those obtained for model M2k. Therefore improving the mesh, including a doubling of the number of points, provides a very substantial improvement in the precision, making these values more acceptable for theoretical modeling.

The middle panel of Fig. 4 presents the differences obtained with models providing fourth-order integration solutions or second-order plus Richardson extrapolation. The global precision here is similar to the previous case or even slightly lower, with differences lower than $0.02 \mu \mathrm{Hz}$. It is interesting to point out that we cannot directly distinguish between a fourth-order integration scheme solution (OSCROX) or a second-order plus Richardson extrapolation (the rest). However, it is noticeable that the GraCo-OSCROX-POSC fall in one group and ADIPLS-NOSC in a second, with a slight difference in the latter case. These two groups are distinguished by the value of $G$ (cf. Table 2), with ADIPLS and NOSC having similar but not identical values. This pattern will be found in other cases also. The LOSC behaviour is discussed in the next paragraph.

Finally in the bottom panel of Fig. 4 a comparison between the different "degrees of freedom" using only the GraCo code are presented. As reference we have used the solution with "degrees of freedom": $X=(\ell=0$, no Richardson extrapolation, $\left.P^{\prime}, r\right)$. For each comparison we have changed only one of these "degrees of freedom" at a time, keeping the rest unchanged (solutions $X^{\prime}$ ). The most prominent effect arises from the use of Richardson extrapolation, which changes the frequencies by nearly $0.8 \mu \mathrm{Hz}$ for the highestorder modes, substantially more than the expected observational accuracies. For model M2k (see Fig. 3), we have similarly found a change of $2 \mu \mathrm{Hz}$, reflecting the smaller number of mesh points. This clearly shows that second-order schemes are inadequate, even for the mesh in M4k, for the computation of high-order acoustic modes; as expected the effect decreases rapidly with decreasing mode order. The use of $r / P$ as integration variable provides small differences, always lower than $0.008 \mu \mathrm{Hz}$. These differences are of the order of those obtained in the top and middle panels. The use of the Lagrangian perturbation to the pressure as variable $(\delta P)$ or the use of the LAWE differential equation provide very similar differences, lower than $0.05 \mu \mathrm{Hz}$, but with an oscillatory pattern. This pattern is very similar to that observed for LOSC, which also uses LAWE to obtain the radial modes. As discussed in Sect. 7.2, this oscillatory pattern arises from an inconsistency in the thermodynamics of model M4k which affects $A^{*}$; solutions of equations that do not depend on $A^{*}$ (i.e., the LAWE or the equations based on $\delta P)$ are insensitive to this effect. Therefore, even for model $\mathrm{M} 4 \mathrm{k}$ and radial modes, the use of different integration procedures can give different values for the oscillation eigenfrequencies that are non-physical in nature. These non-physical sources of differences are mainly some inconsistencies in the equilibrium models (see Sect.7.2) and the lack of mesh 

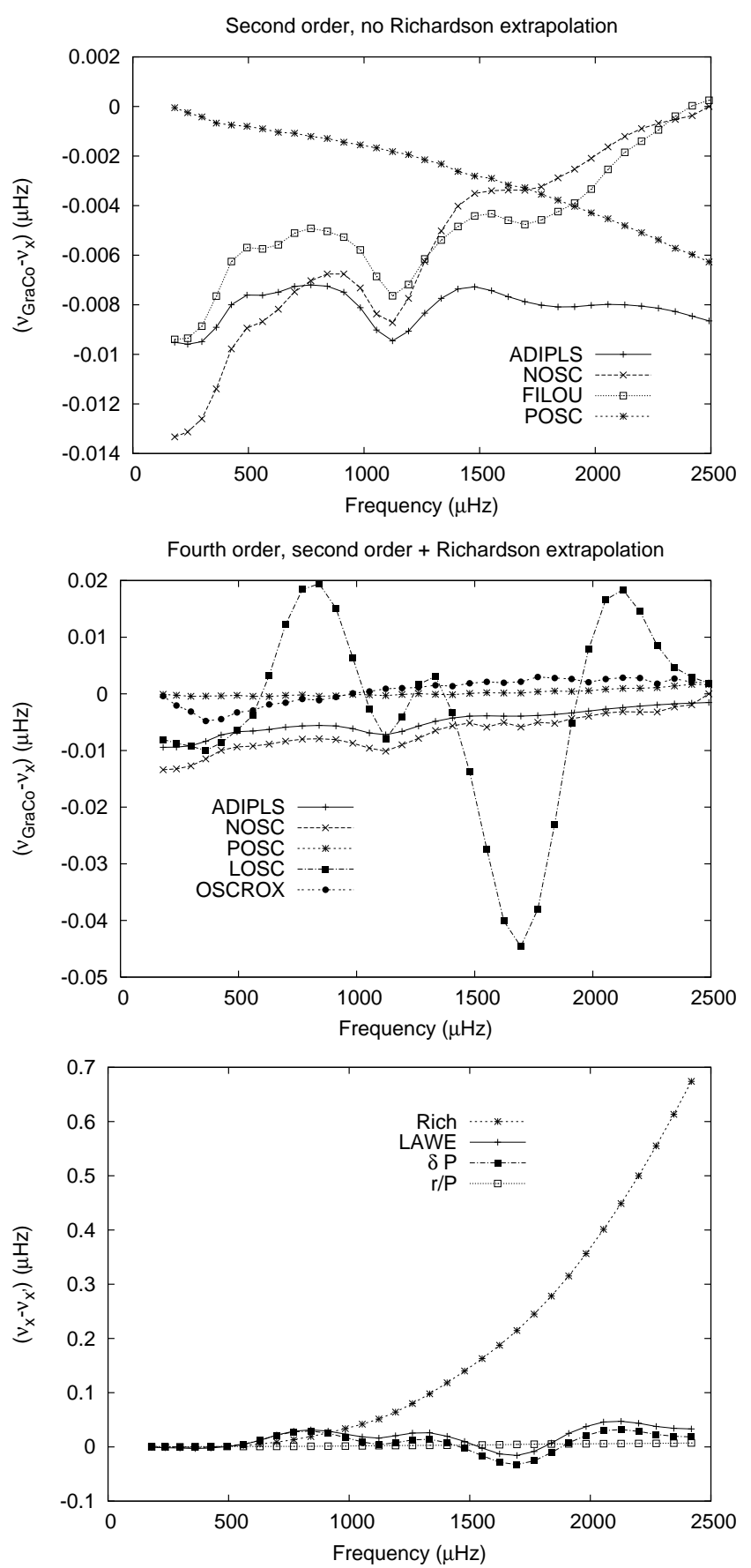

Fig. 4 Frequency comparison (reference line is $\mathrm{GraCo}$ ) for modes with $\ell=0$ as a function of the frequency obtained for the model M4k. In the top panel the models with: second order, no Richardson extrapolation, $r$, are depicted (NOSC-ADIPLS-GraCo-FILOU-POSC). The middle panel presents the differences obtained for models providing fourthorder integration solutions or second-order plus Richardson extrapolation (LOSC-OSCROX-NOSC-ADIPLS-GraCo-POSC). In the bottom panel a comparison of the different "degrees of freedom" only using GraCo is presented. points. But when the same numerical schemes are used, the different codes provide very similar frequencies.

\subsection{Non-radial modes with $\ell=2$}

To illustrate the differences appearing in the case of nonradial modes, the spherical degree $\ell=2$ has been chosen. We have divided the frequency spectrum into three regions: 1 ) Large frequencies $([500,2500] \mu \mathrm{Hz}), 2)$ frequencies around the fundamental radial mode $([80,500] \mu \mathrm{Hz})$, and 3) lowfrequency region $([20,80] \mu \mathrm{Hz})$. In all cases a study similar to that developed in the radial case has been carried out.

For the sake of simplicity the high-frequency differences are not represented since the results are very similar to those presented for the radial case. Only a slightly higher precision is found in this case. The results of LOSC present the same pattern as the radial case, owing to the use of $\delta P$ as eigenfunction in that code (see Sect. 7.2).

Figure 5 shows the results obtained for model M2k when comparing $\ell=2$ frequencies around the fundamental radial mode. The main differences are smaller than in the high-frequency region, corresponding to the low order of the modes and the consequent lesser sensitivity to the number of mesh points. The largest differences are found for two modes of frequency near 345 and $362 \mu \mathrm{Hz}$ showing avoided crossing; these modes have a mixed character, with fairly substantial amplitude in the $\mu$-gradient zone. PULSE and LOSC present differences around $3-4 \mu \mathrm{Hz}$, POSC around $2 \mu \mathrm{Hz}$, OSCROX less than $1 \mu \mathrm{Hz}$, and the rest do not present significant differences for these two modes. That is, the largest differences are found in these codes when using a fourth-order integration scheme or a second-order plus Richardson extrapolation. The values of these differences are larger than the expected precision of the satellite data to come. They clearly reflect the inadequate representation of the $\mu$-gradient zone in $\mathrm{M} 2 \mathrm{k}$, with higher-order schemes being more sensitive to the resulting inconsistency in the model.

In the top panel of Fig. 6 the inter-comparisons of the frequencies $(\ell=2)$ for model M4k, using a second-order scheme without Richardson extrapolation, are depicted for the same frequency range as in Fig. 5. The main differences are two orders of magnitude lower than those obtained for model M2k, and they are also of the same order of magnitude as those obtained for the high-frequency range with M4k. All these differences remain always lower than $0.025 \mu \mathrm{Hz}$. GraCo and POSC are extremely close, while FILOU and ADIPLS provide very similar results, with slightly larger differences for NOSC, by about $0.005 \mu \mathrm{Hz}$, relative to these codes. Thus again the differences are related directly to the different values of $G$. There remain small wiggles for the mixed modes near $350 \mu \mathrm{Hz}$ but reduced more than two orders of magnitude relative to the largest differences for model M2k, reflecting the superior resolution of the critical region in model M4k.

The middle panel of Fig. 6 shows the same inter-comparison as the top panel for codes using a fourth-order scheme 


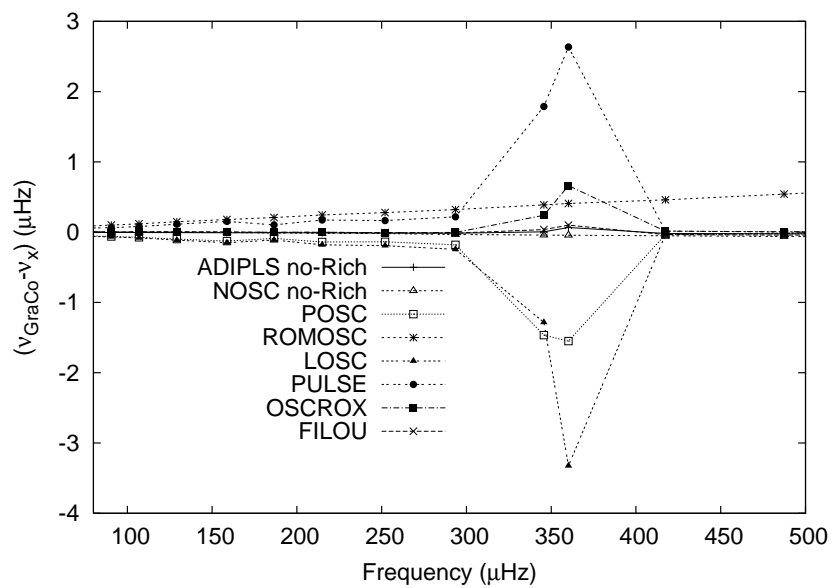

Fig. 5 Frequency comparison (reference line is GraCo) for modes with $\ell=2$ for the model M2k around the fundamental radial mode. ADIPLS and NOSC frequencies have been obtained without using Richardson extrapolation.

or a second-order plus Richardson extrapolation. The precision is similar to the previous case, with differences of the same order of magnitude. We can distinguish two groups of codes providing very similar results: OSCROX-POSC-GraCo and LOSC-ADIPLS-NOSC. This distribution does not depend on the integration scheme, but again reflects the values of $G$. The wiggle of the mixed modes is also very similar to that obtained with a second-order scheme without Richardson extrapolation.

Finally, an inter-comparison of different "degrees of freedom" using only GraCo is presented in order to test the differences obtained for the different choices. This comparison is depicted in the bottom panel of Fig. 6. The use of the Richardson extrapolation is not very important, as expected for these modes of low order, with effects generally smaller than $0.002 \mu \mathrm{Hz}$, although a larger value is present for the mixed modes. Using $\delta P$ as variable gives differences larger than the differences among codes with the same "degrees of freedom". The use of the integration variable $r / P$ does not introduce significant differences.

Compared with model M2k, we find a general reduction of the differences for M4k, the main effects being in the region of mixed modes with an improvement reaching up to three orders of magnitude. This is obviously not a simple consequence of the doubling of the number of mesh points. The main reason is likely the inadequate resolution shown by model M2k in the description of the Brunt-Väisälä frequency in the region close to the boundary of the convective core, which is not present in model M4k. The avoidedcrossing phenomenon and the behaviour of the mixed modes are very sensitive to the detailed treatment of this region, including the effects of semiconvection. Therefore, an accurate description of $N^{2}$ is critical for the oscillation codes in order correctly to obtain eigenfrequencies for modes near an avoided crossing.

The direct frequency inter-comparison ends with the study of the low-frequency region; as above we concentrate on
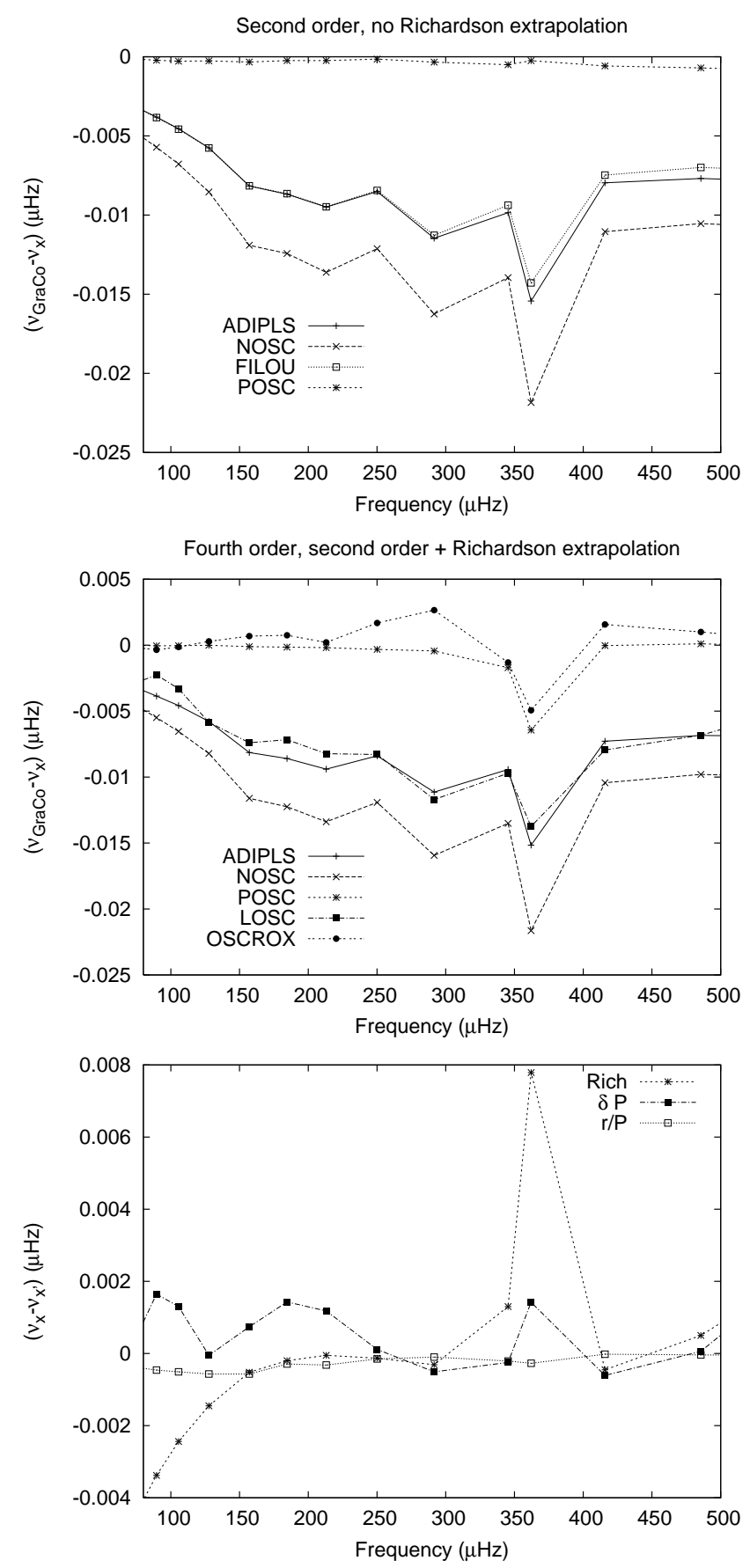

Fig. 6 Frequency inter-comparison (reference line is $\mathrm{GraCo}$ ) for modes with $\ell=2$ as a function of the frequency obtained for the model M4k and for modes around the fundamental radial. In top panel models with: second order, no Richardson extrapolation, and $r$ are depicted (NOSC-ADIPLS-GraCo-FILOU-POSC). Middle panel presents the differences obtained for the models providing fourth-order integration solutions or second-order plus Richardson extrapolation (LOSCOSCROX-NOSC-ADIPLS-GraCo-POSC). In bottom panel an intercomparison for the "different degrees" of freedom only with GraCo is presented. 


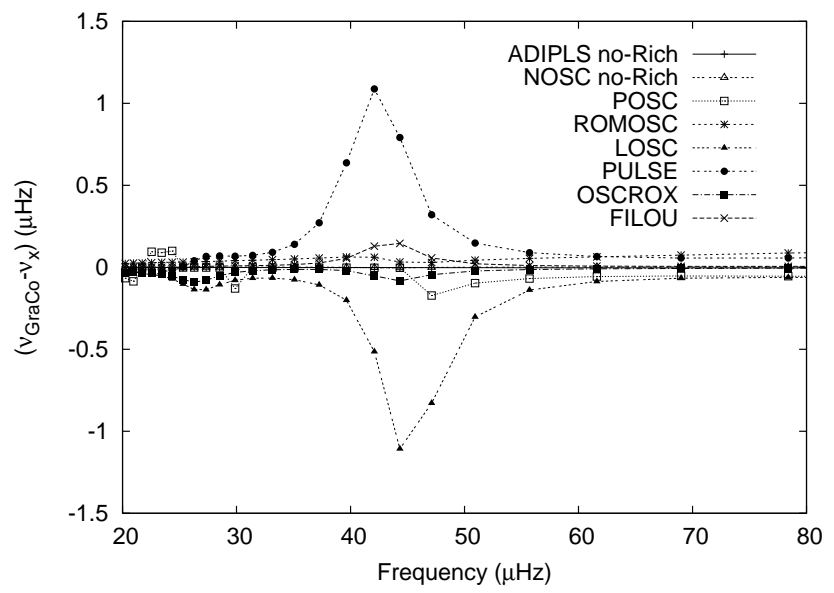

Fig. 7 Frequency comparison (reference line is GraCo) for modes with $\ell=2$ obtained for the model M2k in the low frequency region. ADIPLS and NOSC frequencies have been obtained without using the Richardson extrapolation.

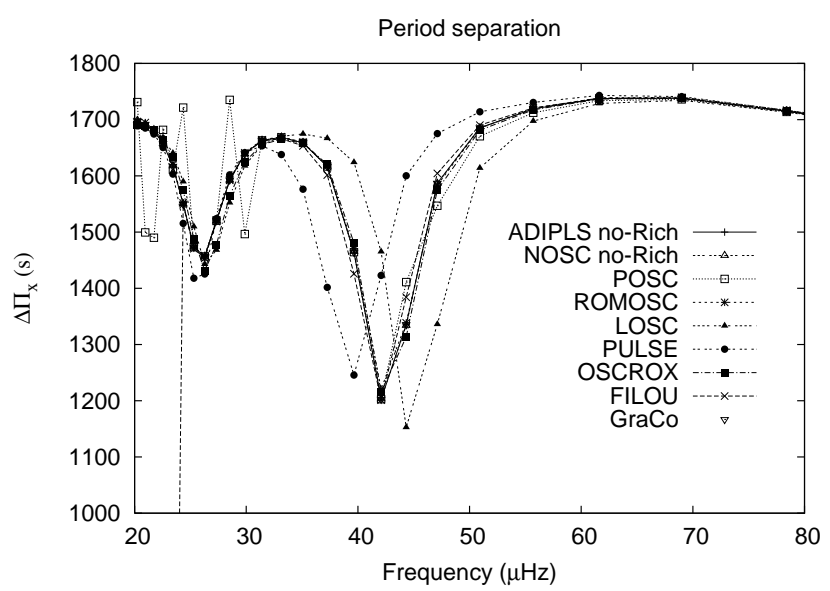

Fig. 8 Period separation as a function of the frequency in the asymptotic g-mode region for model M2k.

modes of degree $\ell=2$. Fig. 7 shows the results obtained for model M2k. In this case the differences are lower than obtained at higher frequencies for this model (cf. Figs 3 and 5). The most surprising behaviour is present in PULSE and LOSC. POSC and OSCROX also present some differences in the same region. To understand the reason for these differences and the region where they appear Fig. 8 shows the period separation $\Delta \Pi$ between adjacent modes. The firstorder asymptotic g-mode theory predicts a constant separation of the periods in this regime for a given $\ell$. However, when the equations are solved numerically, this period spacing presents several minima, and these minima are directly linked with the mode trapping (Brassard et al., 1992b). Fig. 8 shows that the position of one of these minima is the same as the position of the largest differences. The modes in this region have somewhat enhanced amplitudes in the region just outside the convective core. In PULSE and LOSC this apparently happens for modes somewhat different from the remaining codes. This is the origin of the frequency differences. As the mode trapping in this region for these stellar models is related to the Brunt-Väisälä frequency in the $\mu$-gradient zone, the previously mentioned inadequate treatment of this region in model M2k is likely the reason for these differences.

The top panel of Fig. 9 presents inter-comparisons for $\ell=2$ frequencies calculated with a second-order scheme without Richardson extrapolation and for model M4k, for the same frequency range as in Fig. 7. POSC is again extremely close to GraCo, within $2 \times 10^{-4} \mu \mathrm{Hz}$. FILOU and ADIPLS present very similar results, with NOSC showing slightly larger differences, ranging from 0.0005 up to $0.0015 \mu \mathrm{Hz}$ relative to this group. The differences decrease globally as far as the frequency decreases until reaching a magnitude of $0.0015 \mu \mathrm{Hz}$ for the smallest frequency studied here.

The middle panel of Fig. 9 shows the same comparison for codes using a fourth-order scheme or a second-order plus Richardson extrapolation. Once again it can be seen that the precision resembles the results in the previous panel. Here POSC and OSCROX provide very similar results to GraCo. ADIPLS-LOSC-NOSC present small differences among them, with NOSC having a small increasing difference with frequency with respect to the other two. Again the general pattern here, and in the top panel, largely reflects the differences in $G$. In this case we can distinguish the codes using fourthorder integration scheme with its apparently noisy profiles as compared with a solution using second-order integration plus Richardson extrapolation.

In the bottom panel of Fig. 9, an inter-comparison for the different "degrees of freedom" using only GraCo is presented; as in earlier corresponding plots the same value of $G$ is used as in the computation of the equilibrium model. As expected the Richardson extrapolation has a growing influence as the frequency decreases and the mode order increases, with quite substantial differences, compared with the differences between different codes, for the lowestfrequency modes. Thus, with the mesh provided by the evolution calculation the second-order schemes have inadequate numerical precision. In this case, the differences provided by the use of $r / P$ as integration variable are negligible; The use of the Lagrangian perturbation to the pressure $(\delta P)$ gives rise to frequency differences exceeding those obtained between the different codes, at the lowest frequencies; we note, however, that a corresponding comparison between ADIPLS and LOSC does not show this effect which may therefore be particular to the GraCo implementation.

Finally, we want to point out that in the case of model M4k, the large differences appearing in the mode trapping region are not found. Fig. 10 presents the same period separation as Fig. 8 but for this model. All the codes give quite similar results. Two mode trapping regions appear with the same frequency domain as in Fig. 8. As this model does not present any numerical imprecision in the Brunt-Väsälä frequency pattern, the obvious conclusion is that, as for the mixed modes in avoided crossing, any numerical impreci- 

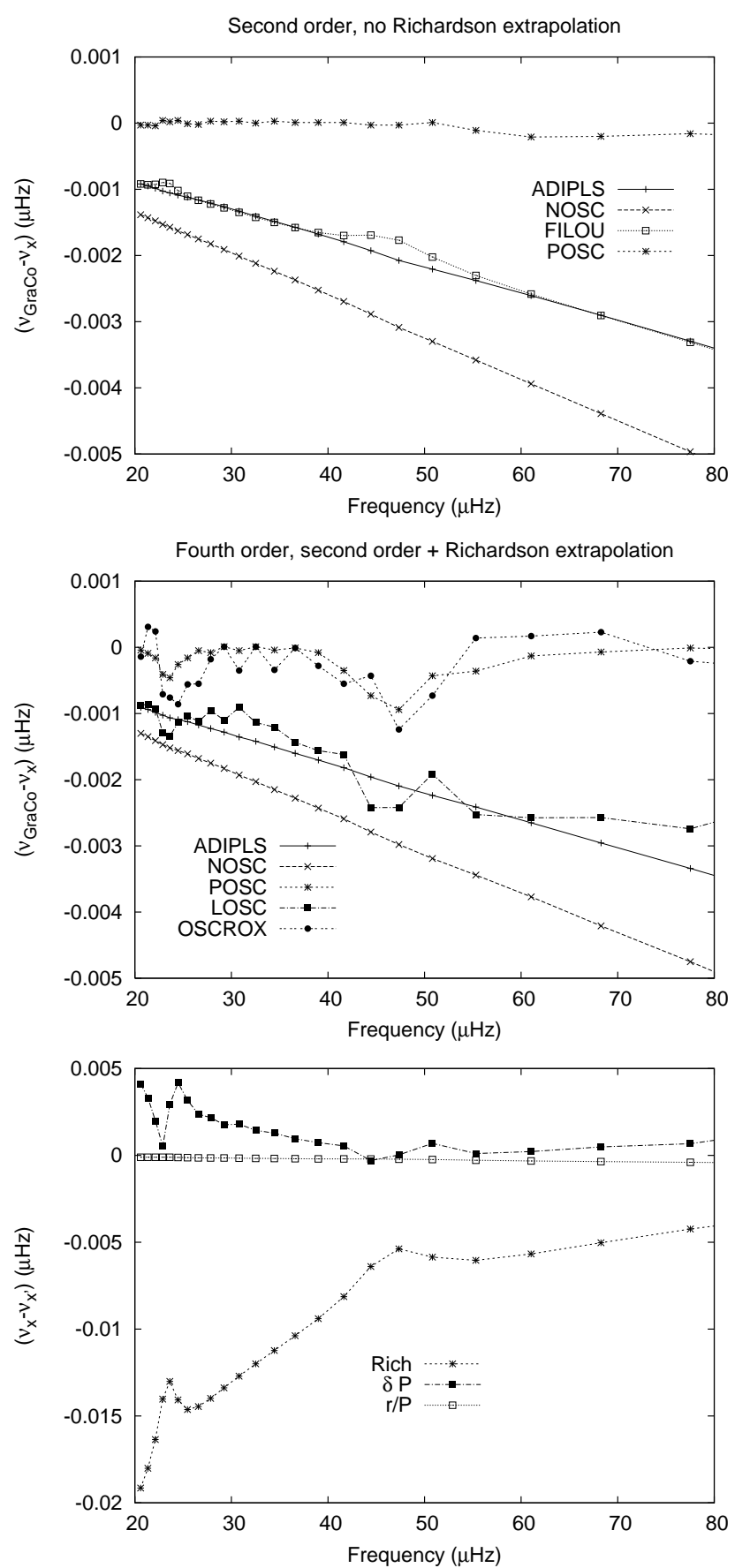

Fig. 9 Frequency comparison (reference line is $\mathrm{GraCo}$ ) for modes with $\ell=2$ as a function of the frequency obtained for the model M4k in the low-frequency region. In the top panel the models with: second order, no Richardson extrapolation, and $r$, are depicted (NOSC-ADIPLSGraCo-FILOU-POSC). The middle panel presents the differences obtained for the models providing fourth-order integration solutions or second-order plus Richardson extrapolation (LOSC-OSCROX-NOSCADIPLS-GraCo-POSC). In the bottom panel an inter-comparison for the different degrees of freedom only using GraCo is presented.

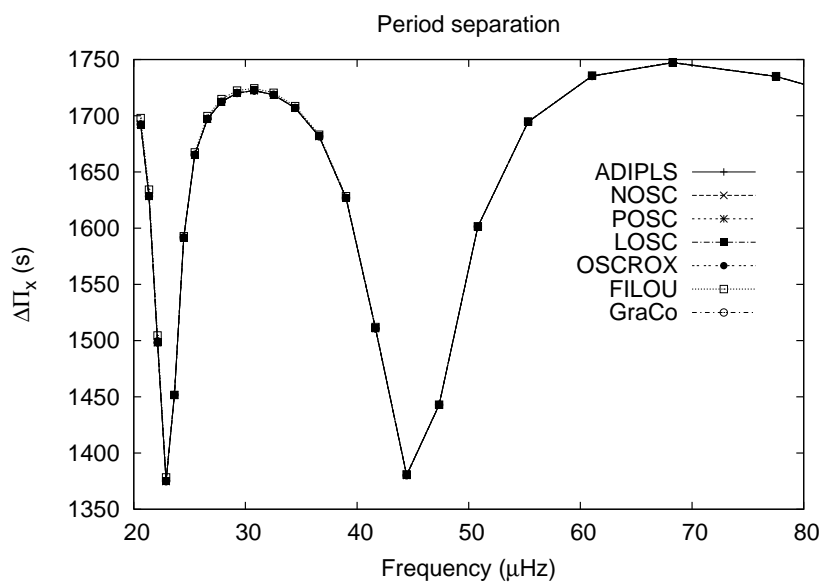

Fig. 10 Period spacing as a function of the frequency in the asymptotic g-mode region for the model M4k.

sion in the description of $N^{2}$ coming from the equilibrium model can give rise to large differences in the frequencies calculated by different oscillation codes for the g-modes trapped in the $\mu$-gradient zone.

\section{Large separations (LS).}

This section is devoted to the asymptotic behaviour of pmodes through the use of the so called "large separations", that is, the difference between two consecutive modes with the same spherical degree $\ell(\Delta=v(n, \ell)-v(n-1, \ell), n$ being the radial order of the mode). The structure of the section is similar to the previous one. We will use the same definitions as in the previous section to study all the frequencies ranges. From now on, the results with the M2k model will not be discussed, since no additional information is found from the further inter-comparisons.

\subsection{Large separation of radial modes.}

Figure 11 shows the results obtained for model M4k. In the top panel differences for the different codes using second order, no Richardson extrapolation, $r$, are depicted. POSC and GraCo are again extremely close. The rest of the codes (ADIPLS-NOSC-FILOU-GraCo) present differences of up to around $0.0015 \mu \mathrm{Hz}$, generally sharing an oscillatory pattern, particularly at relatively low frequency; we have no explanation for this behaviour. However, the effect is evidently small.

In the middle panel of Fig. 11 the LS differences for the different codes, using a fourth-order integration scheme or a second-order plus Richardson extrapolation, are presented. With the exception of LOSC the global behaviour is similar to that obtained without Richardson extrapolation, the NOSCADIPLS-OSCROX-GraCo differences being always lower than $0.002 \mu \mathrm{Hz}$. The pattern of the LOSC differences, presenting 

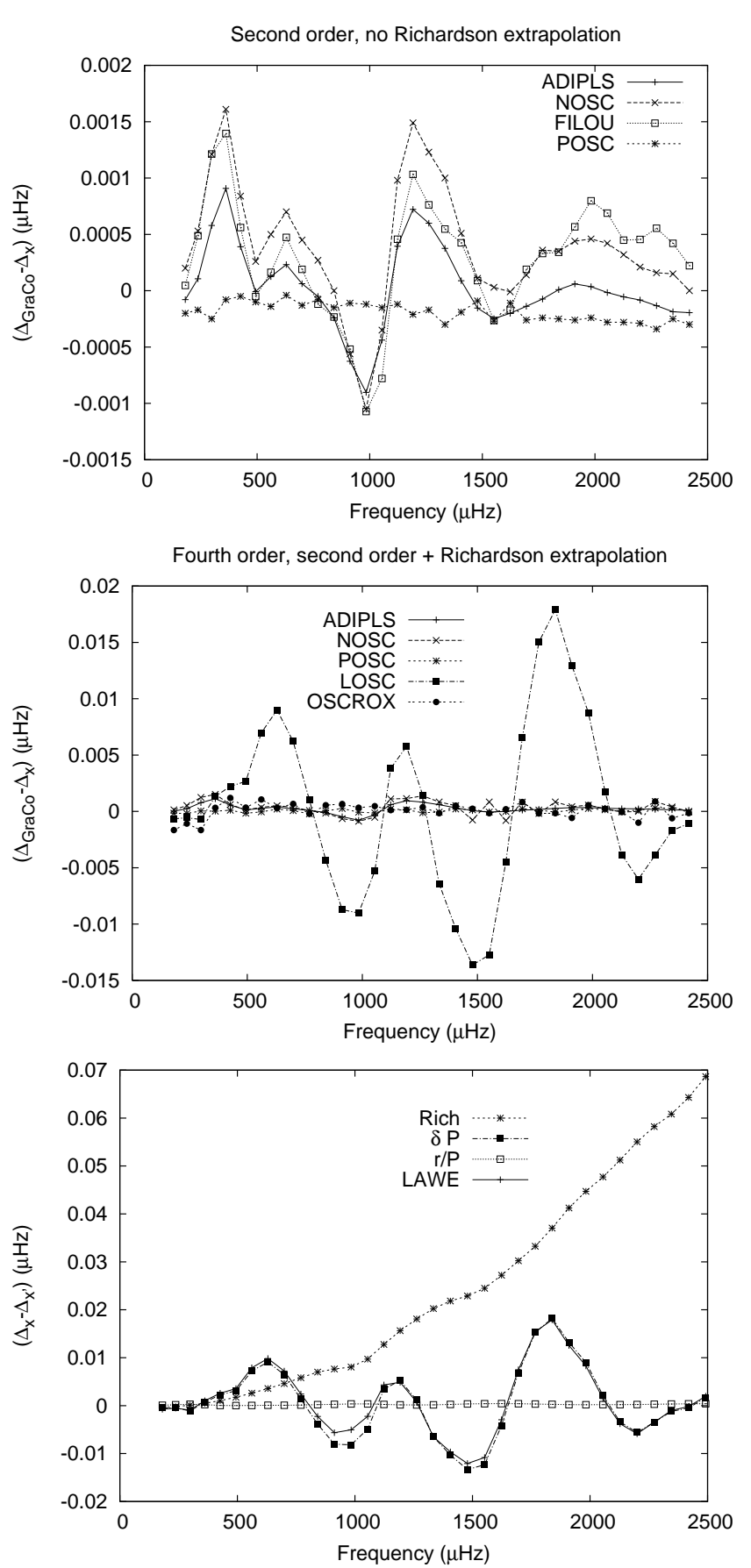

Fig. 11 Large separation inter-comparison (reference line is GraCo) for modes with $\ell=0$ as a function of the frequency calculated for the model M4k. In the top panel the models with second order, no Richardson extrapolation, $r$, are depicted (NOSC-ADIPLS-GraCo-FILOUPOSC). The middle panel presents the differences obtained for the models using fourth-order integration solutions or second-order plus Richardson extrapolation (LOSC-OSCROX-NOSC-ADIPLS-GraCoPOSC). In the bottom panel an inter-comparison for the different "degrees of freedom" using only GraCo is presented. differences one order of magnitude larger than the rest of the codes, is clearly related to the corresponding oscillatory pattern found in Fig. 4, as before it is explained by the use of the LAWE differential equation.

The bottom panel of Fig. 11 shows the LS differences for the radial modes obtained with GraCo and model M4k when different "degrees of freedom" are used. The Richardson extrapolation introduces differences increasing with frequency and mode order, as expected, giving the largest differences, around $0.07 \mu \mathrm{Hz}$. This again emphasizes the inadequacy of the second-order schemes for the highest-order modes, on the M4k mesh. The integration variable $r / P$ gives differences slightly lower than $5 \times 10^{-4} \mu \mathrm{Hz}$, i.e., much smaller than that found for different codes using the same numerical techniques, as depicted in the previous panels. The use of the Lagrangian perturbation to the pressure $\delta P$ and the LAWE differential equation show the same oscillating behaviour and values as those previously observed for the LOSC results. As discussed above, this is related to the inconsistency in $A^{*}$ in model M4k (see Sect.7.2).

\subsection{Non-radial modes with $\ell=2$}

To illustrate the differences appearing in the case of nonradial modes, the spherical degree $\ell=2$ has been arbitrarily chosen. We have divided the frequency spectrum in three regions, like in the direct frequency inter-comparison: 1) highfrequency region, 2) frequencies around the fundamental radial mode and 3) low-frequency region. In all cases, a study similar to that developed in the radial case has been carried out. In the low-frequency region, the more physical period separation is studied, instead of the frequency separation relevant for acoustic modes.

In the first region the results are very similar to those obtained for the radial case; therefore the plots are not presented here. As in the direct frequency inter-comparison case, LOSC also presents an oscillating pattern, owing to the use of $\delta P$ as eigenfunction (see Sect. 7.2). On the other hand, the only noticeable difference, when compared with the radial case in this region, is that for $\ell=2$ the precision among codes using the same integration procedures is slightly higher.

The results obtained with the codes using a second-order scheme, for the modes around the fundamental radial mode, are depicted in the top panel of Fig. 12. POSC remains very close to GraCo, with the other set of codes (FILOU-NOSCADIPLS) extending the oscillatory pattern in the top panel of Fig. 11, with the largest difference being $0.01 \mu \mathrm{Hz}$ for the mixed modes. This set of codes agrees to within differences around $0.001 \mu \mathrm{Hz}$ (slightly larger for NOSC).

The middle panel of Fig. 12 depicts the differences for a fourth-order integration scheme or second-order plus Richardson extrapolation. The precision of the different codes is similar to that given by the second-order scheme without Richardson extrapolation. The maximum difference is lower than $0.08 \mu \mathrm{Hz}$, and most of the codes present differences around $0.005 \mu \mathrm{Hz}$. Once again the largest differences 

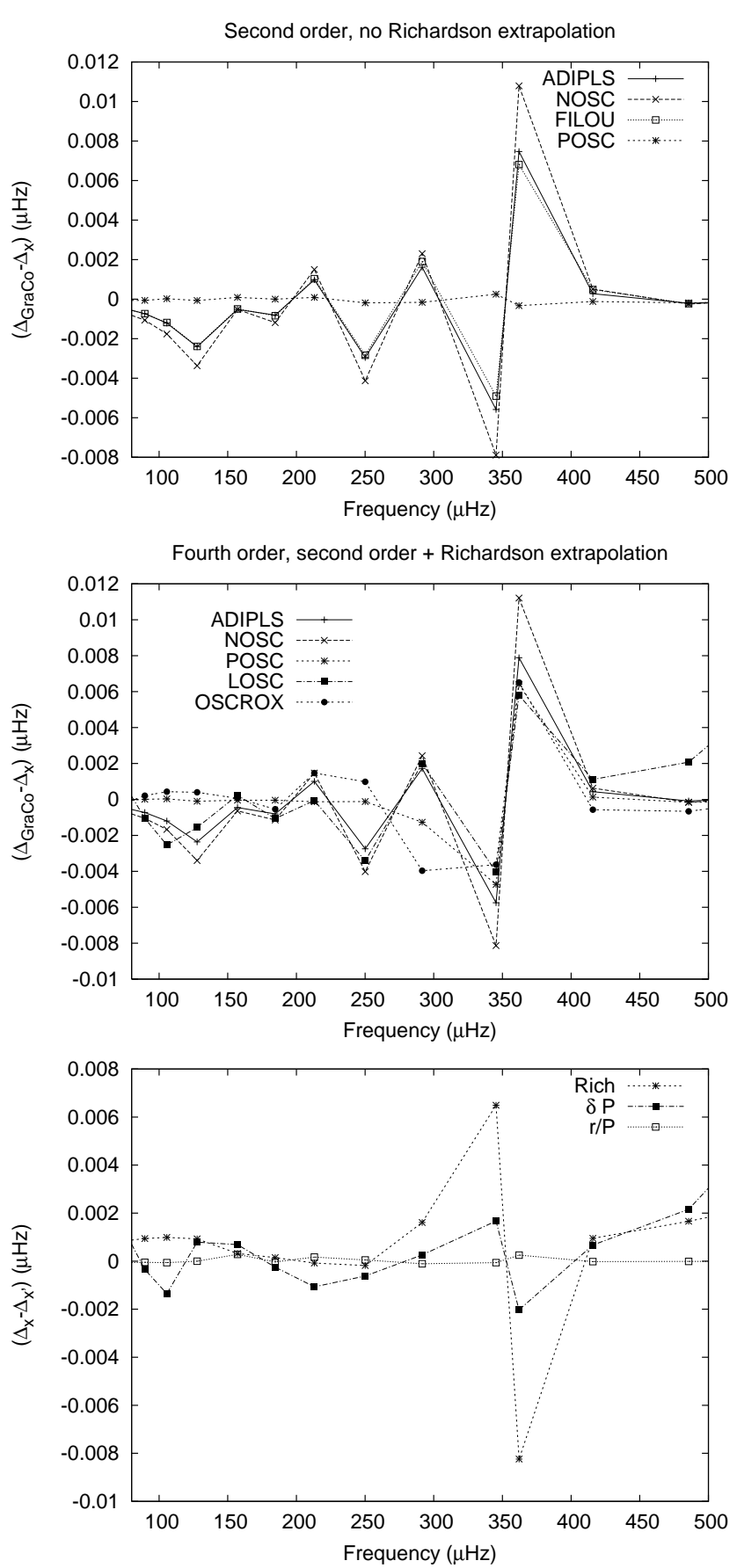

Fig. 12 Large separation inter-comparison (reference line is GraCo) for modes with $\ell=2$ as a function of the frequency calculated for the model M4k and for modes around the fundamental radial one. In the top panel the models with second order, no Richardson extrapolation, $r$, are depicted (NOSC-ADIPLS-GraCo-FILOU-POSC). The middle panel presents the differences obtained for the models using fourthorder integration solutions or second-order plus Richardson extrapolation (LOSC-OSCROX-NOSC-ADIPLS-GraCo-POSC). In the bottom panel an inter-comparison for different "degrees of freedom" using only GraCo is presented. are obtained in the mixed modes. No different behaviours depending on the integration scheme are found.

The bottom panel of Fig. 12 is devoted to the differences obtained with GraCo when different options for the solution of the differential equations are chosen. In this frequency region the replacement of $r$ by $r / P$ as integration variable introduces the smallest differences, one order of magnitude lower than those obtained using the codes with the same "degree of freedom". Using the Lagrangian perturbation to the pressure $(\delta P)$ as eigenfunction causes some difference indicating sensitivity to whether or not $A^{*}$ is used. The Richardson extrapolation introduces differences lower than those found for the different codes, except in the avoided crossing zone of mixed modes, where changes comparable to the largest one are found. Given the rather substantial variations in the bottom panel, and the fact that most codes show the same variation in the top two panels, one might suspect that the dominant source of this variation is in fact in the GraCo results used as reference.

The low-frequency region is studied through the period separation for $\ell=2$ ( $\Delta \Pi$ in seconds), illustrated in Fig. 13 , The top panel of this figure shows the differences found using only codes with a second-order integration scheme. ADIPLS and NOSC show similar shifts of around $0.1 \mathrm{~s}$ relative to GraCo. FILOU is similar at the higher frequencies but shows a small oscillating behaviour in the mode-trapping regions. Finally POSC presents a quite noisy pattern, varying around zero. Again the overall grouping of the differences (ADIPLS-NOSC-FILOU and GraCo-POSC) reflects the different values of $G$.

Results obtained using a fourth-order integration scheme or a second-order plus Richardson extrapolation are compared in the middle panel of Fig. 13 . The values of the differences found in this case are of the same order as in the previous inter-comparison. Here we can distinguish codes using a fourth-order scheme or a second-order plus Richardson extrapolation, because of the apparently random pattern they present, with differences one order of magnitude larger than the main values. The frequency differences obtained with the OSCROX and LOSC results are those presenting a noisy behaviour, when comparing with a second-order plus Richardson extrapolation solution (as GraCo does) as the reference line. POSC also presents some differences in the mode-trapped regions when compared with other codes but using the same integration scheme.

The bottom panel of Fig. 13 shows the differences obtained with the same code ( $\mathrm{GraCo})$ and different choices of the "degrees of freedom". The use of the Richardson extrapolation introduces substantial differences, of the order of seconds, with noticeable wiggles in the two mode-trapping regions. As noted previously this reflects the inadequacy of the second-order schemes for high-order modes. The use of the Lagrangian perturbation to the pressure as variable $(\delta P)$ also introduces substantial differences, particularly around the trapped modes near $22 \mu \mathrm{Hz}$, related to the frequency differences found with GraCo in this region when $\delta P$ is used (cf. Fig. 9]. Using $r / P$ as integration variable gives a small 

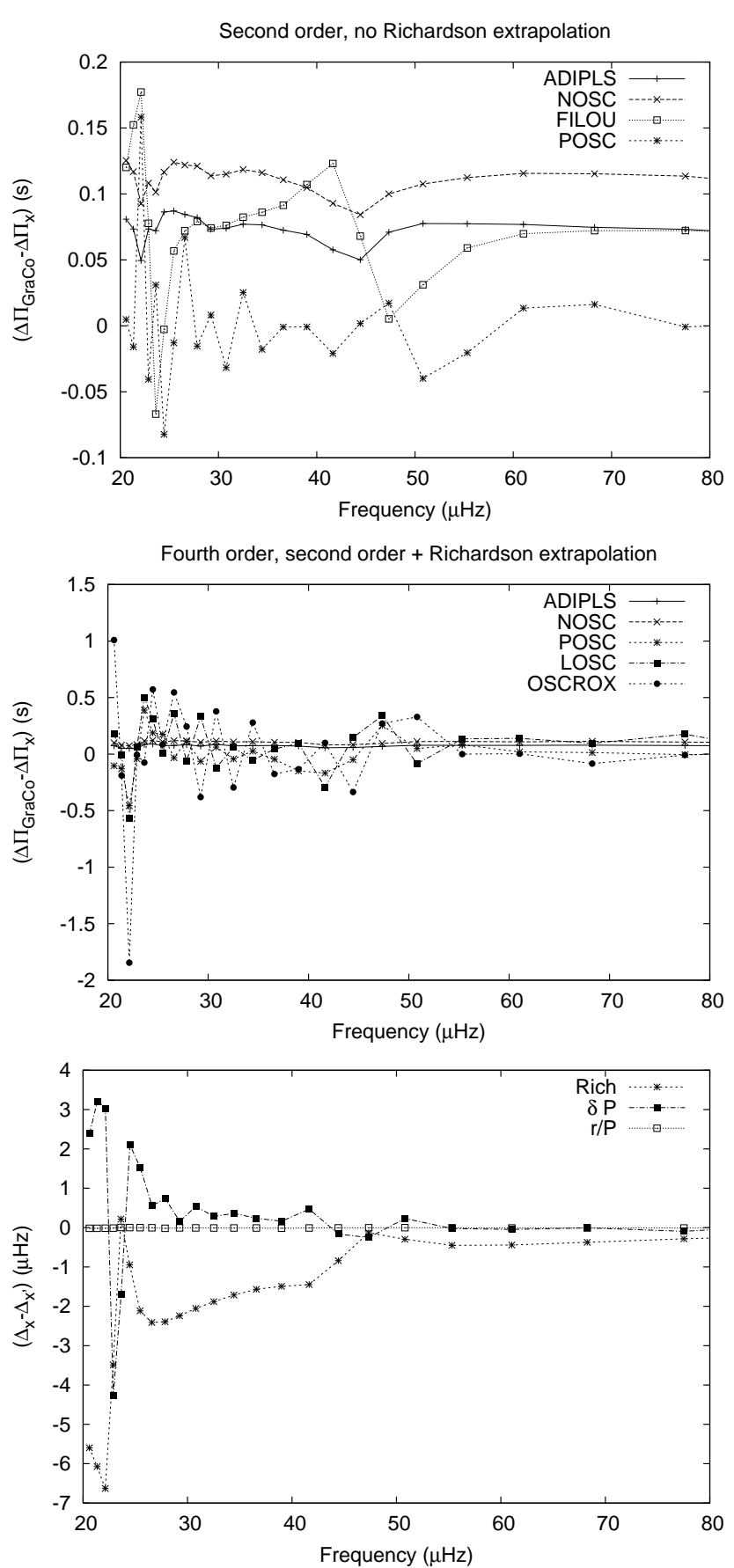

Fig. 13 Period separation inter-comparison (reference line is GraCo) for modes with $\ell=2$ as a function of the frequency calculated for the model M4k in the low-frequency region. In the top panel the models with second order, no Richardson extrapolation, $r$, are depicted (NOSC-ADIPLS-GraCo-FILOU-POSC). The middle panel presents the differences obtained for models using fourth-order integration solutions or second-order plus Richardson extrapolation (LOSCOSCROX-NOSC-ADIPLS-GraCo-POSC). In the bottom panel an inter-comparison for the different choices of "degrees of freedom" using only GraCo is presented. difference when compared with $r$, one order of magnitude lower than the differences found between the codes using the same numerical integration schemes.

\section{Small separations (SS).}

In this section we study the inter-comparisons for the small separations $(\mathrm{SS}) \delta_{\ell \ell^{\prime}} \equiv v(n, \ell)-v(n-1, \ell+2)$. Therefore, two sets of inter-comparisons can be done, one for the $(\ell=0-\ell=2)$ modes and another for the $(\ell=1-\ell=3)$ modes. In this case only the high-frequency region is studied, since this is where this quantity has physical meaning. As in the previous section we concentrate on results for model M4k.

\subsection{Small separations $\delta_{02}$}

The results obtained for the small separation $\delta_{02}$ are presented in Fig. 14. The top panel of this figure shows the differences obtained with the codes solving the set of differential equations with a second-order integration scheme. In the avoided crossing region some wiggles occur related to variations for $\ell=2$, but these wiggles are of the order of magnitude of the differences obtained for the high-frequency region. ADIPLS-POSC-GraCo present similar values for high frequencies and NOSC-FILOU presents differences around $0.004 \mu \mathrm{Hz}$, and always lower than $0.01 \mu \mathrm{Hz}$. These differences are much lower than the expected observational errors for CoRoT.

The middle panel shows the same inter-comparison using a fourth-order integration scheme or a second-order plus Richardson extrapolation. Once again we cannot distinguish the integration scheme used. Just LOSC shows an oscillatory pattern for high frequencies due to the use of LAWE or $\delta P$ (see Sect. 7.2). The order of magnitude of the main differences is the same as those obtained using only a secondorder scheme. The wiggles in the avoided crossing regions are still present, and for high frequencies the differences are all in the range $[-0.001,0.001] \mu \mathrm{Hz}$. ADIPLS and POSC present an almost constant difference with GraCo, NOSC and OSCROX show a small noisy behaviour.

The bottom panel of Fig. 14 presents the SS differences induced in GraCo when different choices of "degrees of freedom" are selected. In this case they are all below, or of the same order of magnitude as, the spread between the different codes illustrated in the middle panel. Interestingly, Richardson extrapolation introduces differences for higher frequencies far smaller than found for the large separation (Fig. 11). The use of the Lagrangian perturbation to the pressure $(\delta P)$ as variable results in the same oscillatory pattern as seen for LOSC in the middle panel. The integration variable $r / P$ gives increasing differences in the range $[0.001,0.003] \mu \mathrm{Hz}$, similar to the Richardson extrapolation, probably reflecting a difference in the sensitivity of radial and nonradial modes to the choice of independent variable in the GraCo code; however, the effect is obviously small. Note that in this case the 

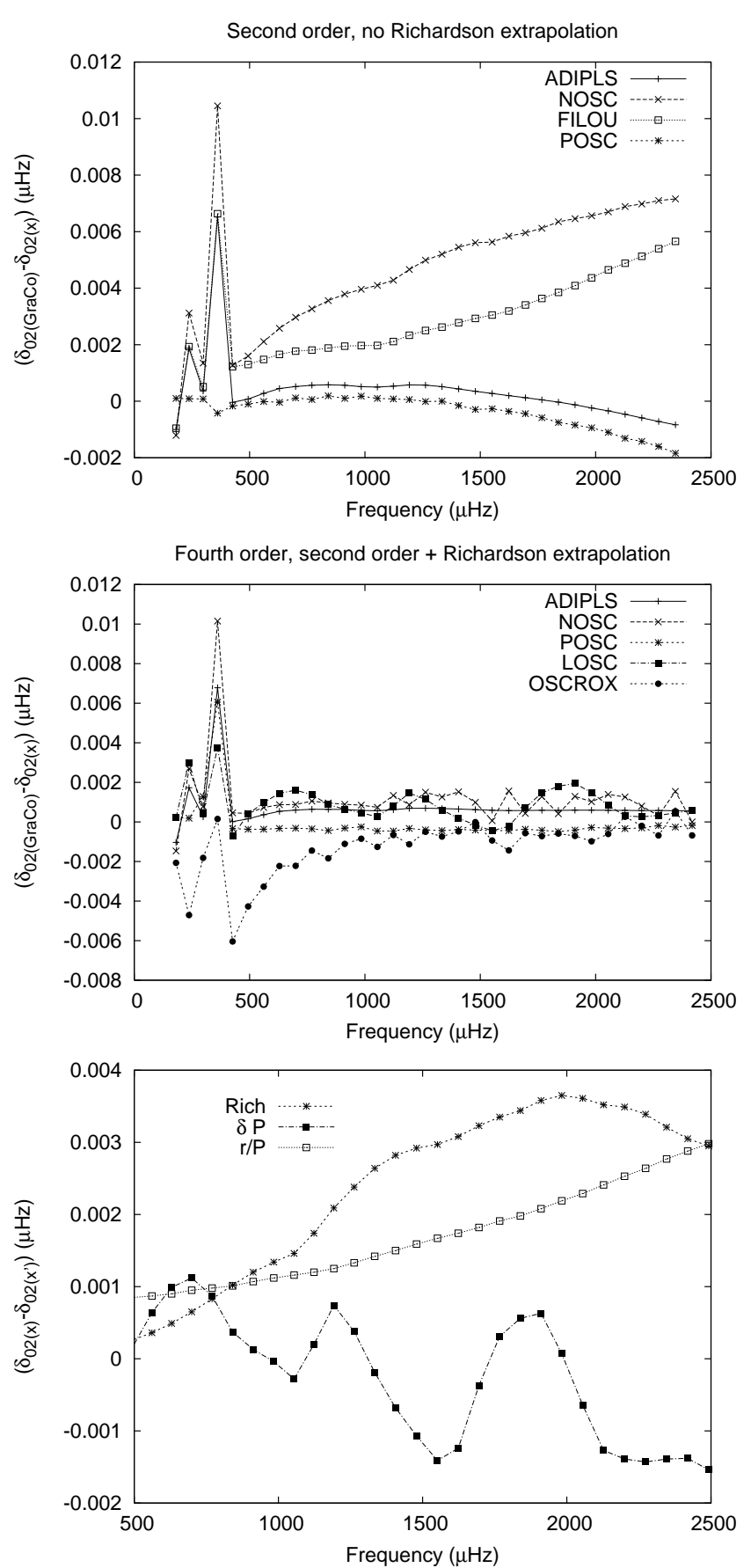

Fig. 14 Small separation inter-comparison (reference line is GraCo) for modes with $\ell=0-2$ as a function of the frequency calculated for the model M4k in the high-frequency region. In the top panel models with second order, no Richardson extrapolation, $r$, are depicted (NOSC-ADIPLS-GraCo-FILOU-POSC). The middle panel presents the differences obtained for models using fourthorder integration schemes or second-order plus Richardson extrapolation (LOSC-OSCROX-NOSC-ADIPLS-GraCo-POSC). In the bottom panel an inter-comparison for different "degrees of freedom" only using GraCo is presented. use of the LAWE for the radial modes, keeping the default "degrees of freedom" (including the use of $P^{\prime}$ ) for $\ell=2$, is hardly meaningful; thus no results are included for LAWE.

\subsection{Small separations $\delta_{13}$}

In Fig. 15 the inter-comparison of $\delta_{13}$ is done for model M4k. The top panel shows the differences given by the codes using a second-order integration scheme. For POSCADIPLS-FILOU-GraCo the differences are between -0.001 and $0.002 \mu \mathrm{Hz}$ and NOSC shows somewhat larger values. In any case the precision is good and the patterns rather smooth.

The codes using a fourth-order integration scheme or a second-order plus Richardson extrapolation have produced the results presented in the middle panel of this figure. The precision is very similar as in the previous panel or even a little higher. LOSC shows the oscillatory pattern already obtained in all the previous inter-comparisons. POSC and ADIPLS present smooth difference profiles when compared with GraCo. On the other hand OSCROX and NOSC give a low noisy profile. Once again the integration scheme used cannot be discriminated.

Finally, the bottom panel presents the effect in $\delta_{13}$ of using different numerical integration schemes. Using Richardson extrapolation gives large differences in the highfrequency region, even larger than the precision of the different codes while, as expected, the effect is small for low-order modes. If the differential equations are solved with $r / P$ as integration variable, an almost increased difference is introduced, always lower than $0.001 \mu \mathrm{Hz}$, i.e., of the order of the precision among most of the codes using the same numerical techniques. The use of the Lagrangian perturbation to the pressure as variable introduces the same oscillatory pattern as was obtained with LOSC.

\section{Computational variations}

\subsection{The influence of the gravitational constant $G$}

As indicated in Table 2 different values of $G$ are used by different codes. In many cases the value differs from the value, $G=6.67232 \times 10^{-8} \mathrm{cgs}$, which was used in the computation of model M4k. As a result, as seen by the pulsation code the equilibrium model is not strictly in hydrostatic equilibrium, thus potentially causing errors in the computed frequencies.

In this section we examine the consequence of such inconsistencies. Thus we test the influence of the choice of a particular gravitational constant $G$, within always the recent experimental values found in the literature. The value of this constant is not very accurately known, and the different values we can find in the literature can have an impact on the frequency calculation of the same order or even larger than the differences studied here. To illustrate this, two extreme values of $G$ found in the literature have been chosen: $G_{1}=6.6716823 \times 10^{-8} \mathrm{cgs}$ (as fixed for Task 1 ), and 

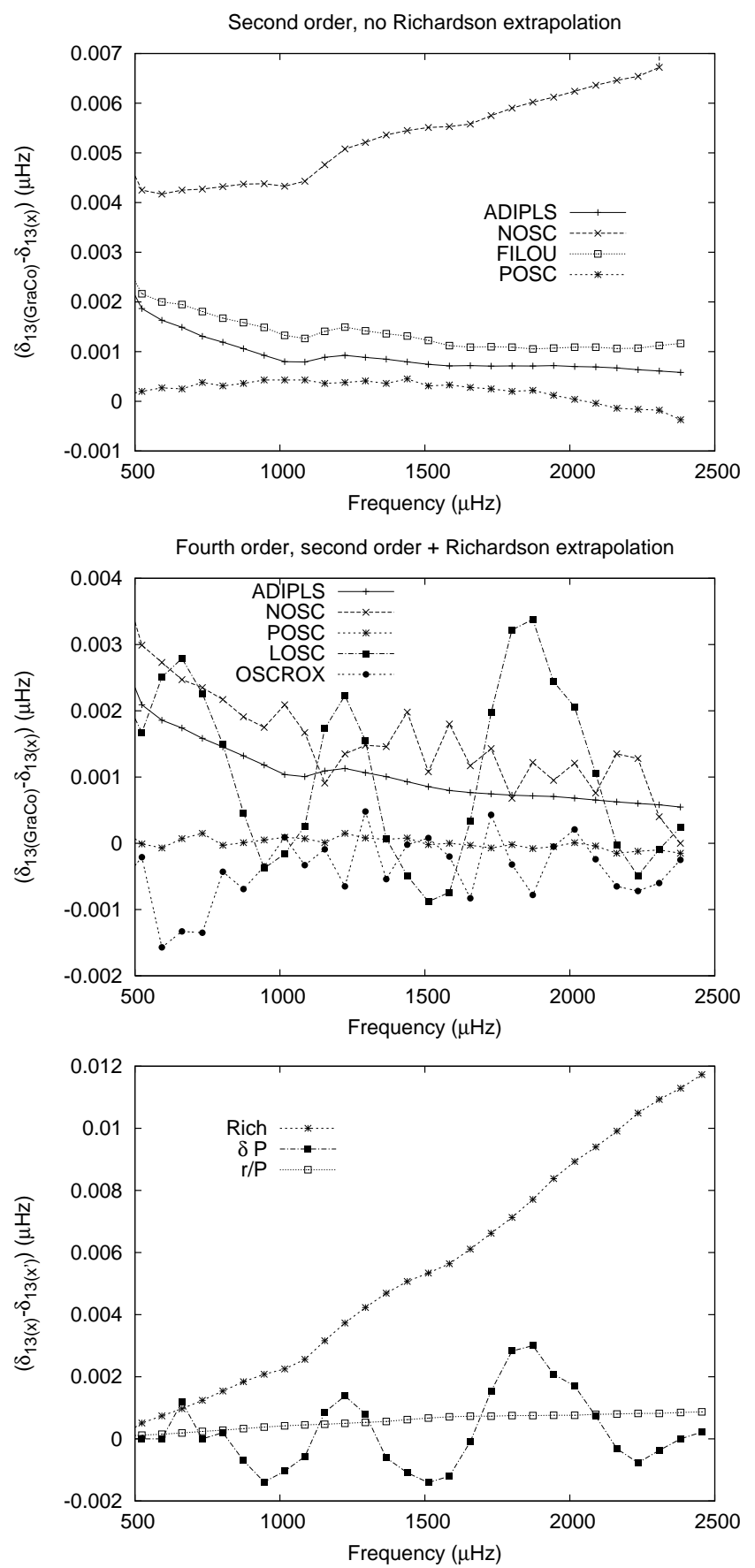

Fig. 15 SS inter-comparison (reference line is $\mathrm{GraCo}$ ) for modes with $\ell=1-3$ as a function of the frequency obtained for model M4k in the high-frequency region. In the top panel the models with second order, no Richardson extrapolation, $r$, are depicted (NOSCADIPLS-GraCo-FILOU-POSC). The middle panel presents the differences calculated for models using fourth-order integration solutions or second-order plus Richardson extrapolation (LOSC-OSCROX-NOSCADIPLS-GraCo-POSC). In the bottom panel an inter-comparison of the different "degrees of freedom" using only GraCo is presented.

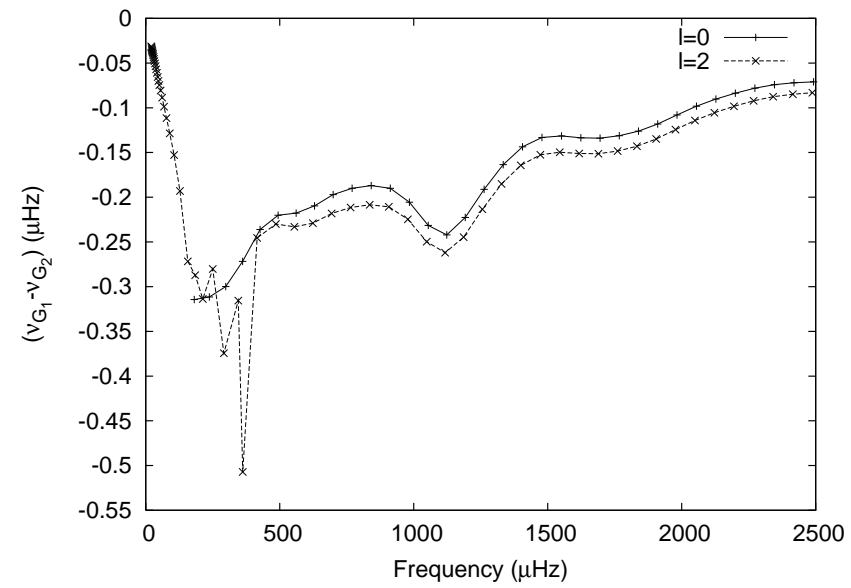

Fig. 16 Frequency comparison of modes with $\ell=0$ and 2 obtained with GraCo, $\left(p^{\prime}, \delta P=0\right.$, no Richardson extrapolation, $\left.r\right)$ and the model M4k when two different values of the gravitational constant $G$ are used.

$G_{2}=6.693 \times 10^{-8} \mathrm{cgs}$ (Fixler et al., 2007), the most recent one, although with a quoted random error of $\pm 0.027 \times 10^{-8}$ and a systematic error of $\pm 0.021 \times 10^{-8} \mathrm{cgs}$ it is consistent with the previous value. The present recommended value of the Committee on Data for Science and Technology (CODATA) can be found in the World Wide Web at physics.nist.gov/constants, and it is closer to that fixed in Task 1. The comparison has been carried out with GraCo at fixed "degrees of freedom" $\left(P^{\prime}, \delta P=0\right.$, no Richardson extrapolation, $r$ ). The differences obtained using both values of $G$ are shown in Fig. 16 for modes with $\ell=0$ and 2. The equilibrium model used is M4k.

Surprisingly, the differences obtained in the region around the fundamental radial mode are much larger than those obtained for the direct frequency inter-comparison for the different codes or for the same code using different integration numerical schemes. As expected for radial modes the largest difference is obtained for the fundamental radial mode. The value of this difference (more than $0.3 \mu \mathrm{Hz}$ in magnitude) is really considerable. In the asymptotic regions the influence of the value of this parameter decreases until reaching values lower than $0.1 \mu \mathrm{Hz}$. Nevertheless, these differences are larger than those obtained by using or not the Richardson extrapolation. The presence of wiggles for $\ell=2$ for the mixed modes is also remarkable.

The relative difference between $G_{1}$ and $G_{2}$ is around $3 \times 10^{-3}$. In contrast, the relative difference between, say, the value chosen for Task 1 and the ADIPLS value is only $5 \times 10^{-5}$. Thus we might expect this difference to cause a frequency difference of order $5 \times 10^{-3} \mu \mathrm{Hz}$. In fact, both the magnitude and shape of the differences between ADIPLS and GraCo in the middle panel of Fig. 4 are consistent with this estimate. In general, as already discussed, it appears that much of the differences between codes using the same numerical scheme results simply from the different values of $G$. Thus, it is obviously important to ensure that consistent 
values of $G$ are used in the evolution and oscillation calculations.

\subsection{The choice of dependent variables and equations}

The most dramatic difference between the different codes is the oscillatory variation shown by the computations using the LAWE or $\delta P$, relative to the reference GraCo results (e.g., Fig. 4). The oscillatory nature and the 'period' of the variation indicated that it reflected a sharp difference in some aspect of the model, located approximately in the region of the second helium ionization zone. After various failed attempts to identify the cause of the variation it transpired that it resulted from a, previously known (e.g., Boothroyd and Sackmann, 2003), inconsistency in the OPAL equation-of-state tables (the original tables of Rogers et al., 1996) used in the computation of model M4k with the ASTEC code.

To understand this we consider the computation of

$A^{*}=\frac{1}{\Gamma_{1}} \frac{\mathrm{d} \ln P}{\mathrm{~d} \ln r}-\frac{\mathrm{d} \ln \rho}{\mathrm{d} \ln r}$.

From the point of view of stellar evolution calculation this is somewhat inconvenient, since the gradient of $\rho$ does not appear directly in the equations of stellar structure. Thus evaluation of $A^{*}$ would involve numerical differentiation of $\rho$. A potentially more convenient formulation is obtained by rewriting the expression in terms of the temperature gradient which is known from the equation of energy transport, given that $\rho$ is a function, known from the equation of state, of pressure, temperature and composition which we characterize solely by the hydrogen abundance $X$. Thus we obtain

$$
\begin{aligned}
A^{*}= & -\left(\frac{\partial \ln \rho}{\partial \ln T}\right)_{P, X}\left(\nabla-\nabla_{\mathrm{ad}}\right) \frac{\mathrm{d} \ln P}{\mathrm{~d} \ln r} \\
& +\left(\frac{\partial \ln \rho}{\partial \ln X}\right)_{P, T} \frac{\mathrm{d} \ln X}{\mathrm{~d} \ln r} ;
\end{aligned}
$$

here, as usual, $\nabla=\mathrm{d} \ln T / \mathrm{d} \ln P$ and $\nabla_{\mathrm{ad}}$ is its adiabatic value. This expression still requires numerical differentiation of $X \sqrt{2}$ but in much of the model $X$ varies little or not at all and hence the term in $\mathrm{d} \ln X / \mathrm{d} \ln r$ makes a limited contribution. Furthermore, in the bulk of convective zones, with homogeneous composition, Eq. 22]accurately reflects the small value of $A^{*}$ resulting from the small value of $\nabla-\nabla_{\mathrm{ad}}$ determined, e.g., from the mixing-length treatment.

The transformation in Eq. 2 obviously assumes that the thermodynamical quantities used are mutually consistent. As noted by Boothroyd and Sackmann (2003), this is not the case of the original OPAL tables provided by Rogers et al. (1996). The effect of this on $A^{*}$ is illustrated in Fig. 17 for model M4k, in terms of the difference between the value

\footnotetext{
2 except if diffusion is taken into account; in that case the gradient of $X$ appears directly in the equations (see, for example, the description of ASTEC by Christensen-Dalsgaard (2007))
}

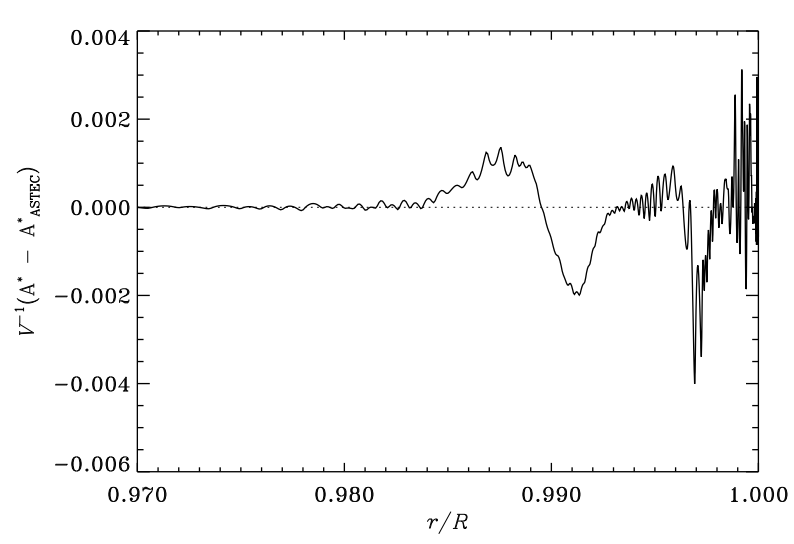

Fig. 17 Differences between $A^{*}$ as evaluated from Eq. 1 and evaluated from Eq. 2] as done in ASTEC, for model M4k, against fractional radius. The differences have been normalized by $V=|\mathrm{d} \ln P / \mathrm{d} \ln r|$.

of $A^{*}$ calculated directly from Eq. 1, using numerical differentiation to evaluate $\mathrm{d} \ln \rho / \mathrm{d} \ln r \sqrt{3}$ and the value, $A_{\mathrm{ASTEC}}^{*}$, evaluated from Eq. 2. There are obvious systematic differences 4 concentrated in the ionization zones of hydrogen and helium. These would indeed affect the oscillations as a 'sharp feature' and hence cause an oscillatory signature in the frequencies, with the 'period' seen in Fig. 4 and elsewhere (e.g., Gough, 1990; Monteiro and Thompson, 2005; Houdek and Gough, 2007).

To test further this interpretation of the results we have recomputed frequencies for model M4k, but replacing $A_{\mathrm{ASTEC}}^{*}$ with $A^{*}$ computed from Eq. 1. For this modified model the differences between ADIPLS and LOSC are smaller by an almost an order of magnitude than the differences illustrated in Fig. 4 and with none of the systematic oscillatory character.

We finally note that transforming between the equations using $P^{\prime}$ and $\delta P$ also depends on the equation of hydrostatic equilibrium and hence on a consistent choice of $G$ in the oscillation equations (cf. Sect.7.1). However, within the actual range of $G$-values used by the different codes this effect is smaller than the effect of the inconsistency in $A^{*}$.

\subsection{The choice of independent variable}

We have found a fairly significant dependence on the $G$ value used by the oscillation codes for the differences of using the integration variables $r$ or $r / P$. Fig. 18 shows this dependence in the case of $\ell=0$. In this figure, the differences between the use of $r$ or $r / P$ with the three different $G$ values presented in Table 2, are depicted. All of them have been obtained using GraCo and M4K. The differences

\footnotetext{
3 at this level of precision it does not matter whether $\mathrm{d} \ln P / \mathrm{d} \ln r$ is calculated from the equation of hydrostatic support or through numerical differentiation.

4 the small-scale rapid variation is probably associated with the interpolation procedure in the equation-of-state tables
} 


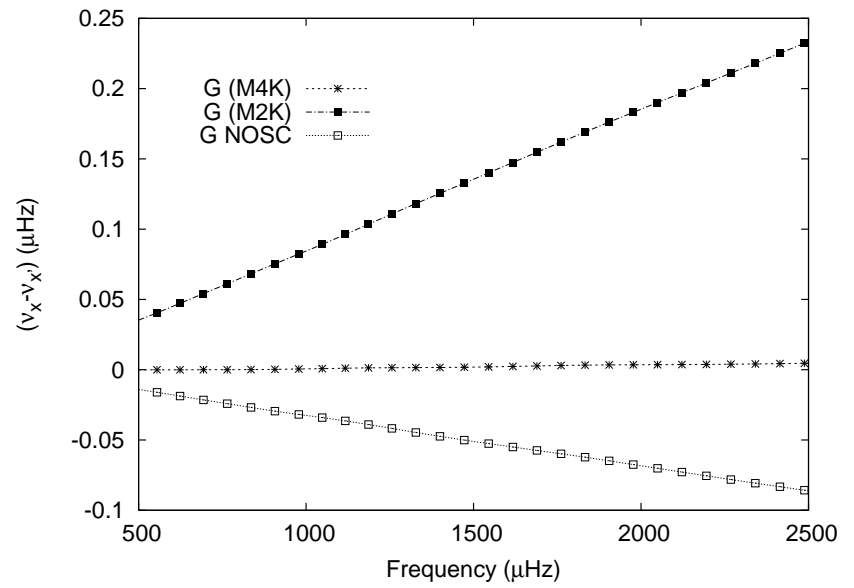

Fig. 18 Differences between the use of $r$ or $r / P$ as integration variable as a function of the mode frequency, for radial modes. Different $G$ values are used in the GraCo oscillation code: $G(\mathrm{M} 4 \mathrm{~K})$ is the $G$ value used to obtain the M4K model, $G(\mathrm{M} 2 \mathrm{~K})$ is the $G$ value used to obtain the M2K model, and $G$ NOSC is the $G$ value used by the NOSC code. $\mathrm{M} 4 \mathrm{~K}$ has been used as equilibrium model for this comparison.

increase with frequency, and their value depend on the difference $G_{e q}-G_{o s c}$, where $G_{x}$ is the $G$ value used to obtain the equilibrium model or the oscillation frequencies, respectively. In fact, the transformation between using $r$ and $r / P$ as independent variables uses the equation of hydrostatic equilibrium and hence the result is obviously sensitive to whether or not a consistent value of $G$ is used. By far the smallest differences are obtained when the same $G$ are used in the equilibrium and oscillation codes.

Even when the same value of $G$ is used, we have found a slight effect of using $r / P$ as independent variable, as implemented in a few of the codes (see also Provost (2007)), instead of $r$; in Fig. 18 the maximum difference is $0.008 \mu \mathrm{Hz}$, as also found in Fig. 4 The origin of choosing $r / P$ seems to be a concern that near the surface, where $r$ varies little between adjacent mesh points, rounding errors in the evaluation of differences in $r$, when representing the differential equations on finite-difference form, could have a significant effect on the results. Given the rapid variation of $P$ in the near-surface layers this problem is obviously avoided if $r / P$ is used instead. While there might well be such problems with using $r$ when variables are represented in singleprecision form (with four-byte reals) it seems unlikely to be a problem when using double-precision variables 5 On the other hand, the present test has been carried out with models transferred in the FGONG format, where variables are given with ten significant digits. Here one cannot exclude that rounding errors might be significant.

To test this JC-D computed frequencies with ADIPLS using both the original binary (double-precision) version of M4k and the version resulting from conversion to FGONG format. The frequency differences between these two cases were always less than $10^{-5} \mu \mathrm{Hz}$, strongly indicating that the

\footnotetext{
5 In contrast, the problem would be severe if the interior mass were used as independent variable.
}

use of the FGONG format is not a concern with the present level of accuracy.

It is obvious that the choice of independent variable also affects the truncation error, i.e., the error in the representation of the differential equations on finite-difference form. This can presumably be the reason for the differences found with GraCo when comparing the use of $r$ and $r / P$ (e.g., Fig. 14), although the effects are small. Further analysis is required to decide which is the more accurate representation.

\section{Conclusions.}

A complete inter-comparison of the frequencies calculated by using a set of oscillation codes has been presented. Nine oscillation codes have been used: ADIPLS, FILOU, NOSC, LOSC, GraCo, OSCROX, PULSE, POSC and LNAWENR. The only free parameter for each code has been the integration numerical scheme. In order to ensure that the same physics has been imposed to all the codes two equilibrium models of a $1.5 M_{\odot}$ star have been supplied, with $4042(\mathrm{M} 4 \mathrm{k})$ and 2172 (M2k) mesh points in their grids, respectively. No remeshing has been allowed. The model M2k presents inadequate numerical resolution in the Brunt-Väisälä frequency close to the boundary of the convective core. The model M4k does not suffer from these problems. In the present paper we have used these models only to show the sensitivities of the oscillation codes to possible numerical inaccuracies in the equilibrium models. A complete study of the accuracies of the different evolutionary codes is provided by Lebreton et al. in this volume.

Several inter-comparisons have been performed: 1) Direct frequency inter-comparison, 2) large separation (LS), 3) small separation (SS), and 4) different experimental values of the gravitational constant $G$.

The main conclusions can be summarized as:

- When codes using the same numerical integration schemes are compared, the general precision obtained for model M4k $(0.02 \mu \mathrm{Hz})$ is higher than the expected precision of the observational data obtained with the CoRoT mission. This precision is always one order of magnitude better or even more when compared with that obtained for model M2k $(0.5 \mu \mathrm{Hz})$. The largest differences are obtained for the high-frequency region.

- The use of a second-order integration scheme plus Richardson extrapolation or a fourth-order integration scheme does not improve the agreement between the codes when compared with the use of a second-order scheme. In the cases here presented it was not possible to distinguish between the use of a fourth-order scheme or Richardson extrapolation, which always give very acceptable consistency for model M4k.

- However, the use of a fourth-order integration scheme (or a second-order plus Richardson extrapolation) introduces differences larger than $0.1 \mu \mathrm{Hz}(1.5 \mu \mathrm{Hz}$ for M2k and $0.8 \mu \mathrm{Hz}$ for $\mathrm{M} 4 \mathrm{k}$ ) compared with a second-order 
scheme, mainly located in the regions of high order (high frequency for $\mathrm{p}$-modes and low frequency for g-modes). This indicates that the second-order schemes have inadequate numerical precision on the mesh provided in M4k and, obviously, even more so on the sparser mesh in M2k.

- For "large separations" the agreement between different comparable codes using model M4k, with every possible numerical scheme is generally better than the expected precision of the data. However, the equation-of-state inconsistency in model M4k (see Sect. 7.2) introduces a signature that it potentially significant. The use of the Richardson extrapolation together with a second-order integration scheme, or a fourth-order scheme, gives differences for high-order $\mathrm{p}$ - and g-modes relative to the use of only a second-order scheme that are comparable to the expected observational accuracies, indicating that the accuracy of the latter scheme is inadequate.

- The "small separations" present differences for model M4k lower than the expected observational accuracies everywhere, although the inconsistency in M4k has a noticeable effect. Use of Richardson extrapolation has a potentially significant effect, indicating that the precision of second-order schemes may be inadequate.

- The use of $r / P$ as integration variable instead of $r$ has a small influence when a consistent value of $G$ is used. However, an inconsistency between the $G$ values used to obtain the equilibrium model and the oscillation frequencies can give substantial differences between the use of these integration variables, over the range of $G$ values in Table 2.

- For model M2k, the mixed modes near the avoided crossing present large differences, reaching even up to $4 \mu \mathrm{Hz}$. These differences are not present when model $\mathrm{M} 4 \mathrm{k}$ is used. The problems with $N^{2}$ in M2k are undoubtedly the main source of this difference. The same behaviour is found for the trapped g-modes. Therefore, the correct numerical description of $N^{2}$ is critical for the value of the frequencies of these trapped modes or in avoided crossing.

- The use of $\delta P$ or $P^{\prime}$ as eigenfunction, or the solution of the LAWE, may have a significant effect if the equilibrium model is based on thermodynamic quantities that are not internally consistent, as is the case for the OPAL tables used to calculate model M4k. Further effects arise when there are problems with the Brunt-Väisälä frequency (as for model M2k); here a different choice can change the frequencies of some modes since $N^{2}$ does not appear in the differential equations when $\delta P$ or the LAWE are used and it does when using $P^{\prime}$.

- The value of the gravitational constant $G$ in the oscillation calculations can introduce non-negligible differences as well, if it is not the same as the value used in the equilibrium model. When the two extreme values found in the literature are used, such inconsistency yields differences in the range $[-0.35,-0.08] \mu \mathrm{Hz}$ for model M4k, larger than those obtained when different numer- ical integration schemes are used. Differences between the values of $G$ actually used by the different codes, although less extreme, account for much of the difference between the computed frequencies.

Therefore, for a proper pulsational study, we require that the number of mesh points and their distribution must be such as to yield an equilibrium model that satisfies the dynamical equations with sufficient accuracy also in the regions of the star where the physical quantities present rapid variations (e.g., the outer layers and $\mu$-gradient zones). In addition, the mesh used in the pulsation calculation must properly resolve the eigenfunctions of the highest-order modes considered. In the present case model M4k, with 4042 points, appears to satisfy these conditions although the use of a fourth-order integration scheme, or a second-order scheme and Richardson extrapolation, is still needed in the oscillation calculation; these higher-order schemes give significant improvements compared with the use of a simple second-order scheme. The use of a second-order integration plus Richardson extrapolation scheme is not distinguishable, in accuracy and precision, from the use of a fourth-order integration scheme. A correct physical and numerical description of the Brunt-Väisälä frequency is essential when $P^{\prime}$ is used as eigenfunction; in particular, inconsistencies in the equation of state can have serious effects on the frequencies. Inconsistency between the value of $G$ used in the oscillation calculation and the value used to compute the equilibrium model, within the range of the different values of $G$ found in the literature, may lead to substantial errors in the computed frequencies.

We note that the situation is somewhat different if consistent values of $G$ are used in the evolution and oscillation calculations. Then the effect on the frequencies is approximately given, according to homology arguments, as a scaling by $(G M)^{1 / 2}$. However, since the product $G M_{\odot}$ is known extremely precisely from planetary motion in the solar system, any variation in $G$ should be reflected in a corresponding change in the assumed value of $M_{\odot}$. If this is the case, and if the model is characterized by a given value of $M / M_{\odot}$ (as is typically the case) the effect on the frequencies of changes in $G$ are very small (see also Christensen-Dalsgaard et al., 2005).

In further tests more care is required to secure the full consistency of the models: a consistent equation of state should be used, and the value of $G$ should obviously be the same in the equilibrium-model and the pulsation calculations; indeed, this strongly argues for including the value of $G$ as one of the parameters in the model file. The main conclusion of this extensive investigation, however, it positive: with a properly resolved equilibrium model the broad range of oscillation codes likely to be involved in the asteroseismic analysis of data from CoRoT and other major upcoming projects generally give consistent results, well within the expected errors of the observations. Thus, although the remaining problems in the calculation evidently require attention, we can be reasonably confident in our ability to compute frequencies of given models and hence in the inferences 
concerning stellar structure drawn from comparing the computed frequencies with the observations.

Acknowledgements This work was supported by the Spanish PNE under Project number ESP 2004-03855-C03-C01, and by the European Helio- and Asteroseismology Network (HELAS), a major international collaboration funded by the European Commission's Sixth Framework Programme. A.M. and J.M. acknowledge financial support from the Belgian Science Policy Office (BELSPO) in the frame of the ESA PRODEX 8 program (contract C90199). MJPFGM is supported in part by FCT and FEDER (POCI2010) through projects POCI/CTEAST/57610/2004 and POCI/V.5/B0094/2005.

\section{References}

Baglin, A., Auvergne, M., Barge, P., Deleuil, M., Catala, C., Michel, E., Weiss, W., and the COROT team: Scientific objectives for a minisat: COROT. ESA SP-1306, 33-37 (2006)

Boothroyd, A. I. and Sackmann, I.-J.: Our Sun. IV. The standard model and helioseismology: consequences of uncertainties in input physics and in observed solar parameters. ApJ 583, 1004-1023 (2003)

Boury, A., Gabriel, M., Noels, A., Scuflaire, R. Ledoux, P.: Vibrational instability of a 1 solar mass star towards nonradial oscillations. A\&A 41, 279-285 (1975)

Brassard, P., Fontaine, G., Wesemael, F., Hansen, C. J.: Adiabatic properties of pulsating DA white dwarfs. II - Mode trapping in compositionally stratified models. ApJS 80, 369-401 (1992b)

Brassard, P., Charpinet, S.: PULSE: A finite element code for solving adiabatic nonradial pulsation equations. Ap\&SS this volume (2007)

Christensen-Dalsgaard, J.: ADIPLS - the Aarhus adiabatic oscillation package. Ap\&SS - this volume (2007)

Christensen-Dalsgaard, J.: ASTEC - the Aarhus STellar Evolution Code. Ap\&SS - this volume (2007)

Christensen-Dalsgaard, J. \& Berthomieu, G.: Theory of solar oscillations. In Solar interior and atmosphere, (Eds) Cox, A. N., Livingston, W. C. \& Matthews, M., Space Science Series, University of Arizona Press, p. 401-478, (1991)

Christensen-Dalsgaard, J. \& Mullan, D. J.: Accurate frequencies of polytropic models. MNRAS 270, 921-935 (1994)

Christensen-Dalsgaard, J., Di Mauro, M. P., Schlattl, H. \& Weiss, A.: On helioseismic tests of basic physics. MNRAS 356, 587 - 595 (2005)

Christensen-Dalsgaard, J., Arentoft, T., Brown, T. M., Gilliland, R. L., Kjeldsen, H., Borucki, W. J. \& Koch, D.: Asteroseismology with the Kepler mission. In Proc. Vienna Workshop on the Future of Asteroseismology, (Eds) G. Handler \& G. Houdek, CoAst 150, 350-356 (2007)

Fixler, J.B, Foster, G.T. McGuirk, J.M., Kasevich, M.A.: Atom Interferometer Measurement of the Newtonian Constant of Gravity. Science 315, 74-77 (2007)
Gough, D. O.: Comments on helioseismic inference. Progress of seismology of the sun and stars, (Eds) Osaki, Y. \& Shibahashi, H., Springer - Berlin, Lecture Notes in Physics, vol. 367, 283 - 318 (1990)

Houdek, G. \& Gough, D. O.: An asteroseismic signature of helium ionization. MNRAS 375, 861 - 880 (2007)

Monteiro, M.J.P.F.G.: POSC - The Porto Oscillations Code. Ap\&SS - this volume (2007)

Monteiro, M. J. P. F. G. \& Thompson, M. J.: Seismic analysis of the second ionization region of helium in the Sun I. Sensitivity study and methodology. MNRAS 361, 1187 - 1196 (2005)

Morel, P., Lebreton, Y.: CESAM: Code d'Evolution Stellaire Adaptatif et Modulaire. Ap\&SS - this volume (2007)

Moya, A., Garrido, R.: Granada oscillation Code (GraCo). Ap\&SS - this volume (2007)

Provost, J.: NOSC: Nice Oscillations Code. Ap\&SS - this volume (2007)

Rogers, F. J., Swenson, F. J. \& Iglesias, C. A.: OPAL Equation-of-State Tables for Astrophysical Applications. ApJ 456, 902 - 908 (1996)

Roxburgh, I.W.: The OSCROX stellar oscillation code. Ap\&SS - this volume (2007)

Scuflaire, R., Montalbán, J., Théado, S., et al.: The Liège Oscillations Code. Ap\&SS - this volume (2007)

Shibahashi, H. and Osaki, Y.: Theoretical Eigenfrequencies of Solar Oscillations of Low Harmonic Degree $l$ in FiveMinute Range. PASJ 33, 713-719 (1981)

Suarez, J.C., Goupil, M.J.: FILOU Oscillation Code. Ap\&SS - this volume (2007)

Suran, M.: LNAWENR - Linear Nonadiabatic Nonradial Waves. Ap\&SS - this volume (2007) 